NASA Technical Memorandum 101995

SAE Paper No. 89-0332

\title{
Engineering Study of the Rotary-Vee Engine Concept
}

Edward A. Willis

National Aeronautics and Space Administration

Lewis Research Center

Cleveland, Ohio

Timothy A. Bartrand

Sverdrup Technology, Inc.

NASA Lewis Research Center Group

Cleveland, Ohio

and

John E. Beard

National Aeronautics and Space Administration

Lewis Research Center

Cleveland, Ohio

Presented at the

1989 Annual Congress and Exposition

sponsored by the Society of Automotive Engineers

Detroit, Michigan, February 27-March 3, 1989

\section{N/SA}

$\begin{array}{lll}\text { (NASA-TM-101995) } & \text { ENGINEERING STODY OF THE } \\ \text { ROTARY-VEE ENGINE } & \text { CONCEPT } \\ \text { Research Center) } & 38 \mathrm{p} & \end{array}$

N89-26007

Unclas

G3/07 0222716 


\title{
ENGINEERING STUDY ON THE ROTARY-VEE ENGINE CONCEPT
}

\author{
E.A. Willis \\ National Aeronautics and Space Administration \\ Lewis Research Center \\ Cleveland, Ohio 44135 \\ T.A. Bartrand \\ Sverdrup Technology, Inc. \\ NASA Lewis Research Center Group \\ Cleveland, Ohio 44135 \\ and \\ J.E. Beard* \\ National Aeronautics and Space Administration \\ Lewis Research Center \\ Cleveland, Ohio 44135
}

\begin{abstract}
This paper provides a review of the applicable thermodynamic cycle and performance considerations when the rotary-vee mechanism is used as an internal combustion (I. C.) heat engine. Included is a simplified kinematic analysis and studies of the effects of design parameters on the critical pressures, torques and parasitic losses. A discussion of the principal findings is presented.
\end{abstract}

\section{SUMMARY}

The rotary-vee is an unusual two-stroke internal combustion (I. C.) engine which incorporates many small cylinders in a pair of rotating cylinder blocks. Several development projects have been launched since about 1970 to capture the perceived benefits of this arrangement. These activities, however, did not result in sufficient technical data to conduct an objective engineering evaluation. This report presents a new analytical computer program which models the thermodynamics, flow and combustion processes and parasitic losses of the rotary-vee, but does not treat structural, durability or reliability considerations.

The simulation results indicate that the rotary-vee engine concept is thermodynamically feasible, but has no clear advantage over a generic, state-of-the-art two-stroke engine. By comparison, the rotary-vee appears to offer a tradeoff, with a possibly higher power density but a lower efficiency. With this tradeoff, the rotary-vee may be most suitable for specialized applications where size and weight are more important than fuel economy.

Advanced technologies such as ceramic heat barrier coatings could be applied to the rotary-vee in hopes of raising its efficiency. But, as is the case with other I. C. engines,

*NASA Summer Faculty Fellow. Present address: Dept. of Mech. Engr., Louisiana State University, Baton Rouge, Louisiana 70803. 
ceramic coatings offer little improvement unless accompanied by an auxiliary, bottoming cycle device to recover exhaust heat energy. In any case, such technologies would benefit alternative I. C. engines about equally and so should not affect the comparisons made herein.

\section{BACKGROUND}

The rotary-vee mechanism was originally of interest as an angle-drive, i.e., a machine element for transmitting torque around a corner. Due to its volume changing ability, however, it can also be used as a positive displacement engine, pump or compressor. Figure 1 shows a small, hand-operated rotary-vee model with two of its four pistons removed for clarity. In its simplest form, it consists of two rotating cylinder blocks with cylinder bores parallel to the axis. Each corresponding pair of cylinder bores is connected by a double-ended, free-floating vee-piston in the shape of a bent rod. The rotation of the cylinder blocks causes the centers of the vee-pistons to orbit around an oval path, while the pistons themselves do not rotate (i.e., they maintain a fixed orientation in inertial space). Piston reciprocation is developed as a relative motion between the rotating cylinder blocks and the orbiting pistons. Torques resulting from pressure, inertia or friction forces, or external loads, are transmitted by side reactions of the vee-pistons against the cylinder bores.

Figures 2, 3 and 4 illustrate the different phases of a rotary-vee engine as a typical working chamber progresses from minimum to maximum volume. In Figure 2, the piston is at top dead center (TDC) relative to the cylinder. In Figure 3, the piston and cylinder have moved $120^{\circ}$ into the expansion cycle. In Figure 4 , they have moved $180^{\circ}$ to the bottom dead center (BDC) position, at which point the working chamber has the maximum volume. In the next $180^{\circ}$, the piston moves back up to its minimum volume or TDC position. Thus, with the addition of suitable valves or ports, the device can be used as either a two-stroke or four-stroke engine, or alternatively as a pump or compressor.

Based on this mechanism, the rotary-vee has evolved into an unusual two-stroke internalcombustion (I. C.) engine which incorporates many small cylinders within its rotating cylinder blocks. This arrangement has been perceived to offer several potential advantages, such as small size, high efficiency, simplicity, low weight and cost and inherent balance. Several projects have been launched, since about 1970, to develop an engine with these actual characteristics. The earliest pertinent references are popular articles on the Bricklin-Turner engine that appeared in the mid 1970's, $(1)^{*},(2)$. Several prototypes were built, but due to technical and financial problems the Bricklin-Turner project was eventually discontinued. Sullivan (3) then took up the development and in 1984 built and operated an improved prototype. Since then he has continued these efforts (4), aiming towards ultra-light aircraft, small drones and related applications in the sub- $100 \mathrm{hp}$ class.

Despite optimistic media reports (3), (4), these activities have not resulted in enough technical data to support a broad, objective engineering evaluation of the potential and applicability of the rotary-vee engine concept. Although one dynamometer test report was published in 1977 (5), it was quite brief and did not include fuel economy or emissions data. Since then, no further experimental or analytical results on the concept appear to have been published. Until adequate data are in hand, it must be agreed that the potential merits of this engine have not been clearly defined or demonstrated. By contrast, the Orbital two-stroke engine (6), (7), which combines a conventional crank piston engine with direct cylinder fuel injection, has

\footnotetext{
* Numbers in parentheses designate references at end of paper.
} 
been extensively tested by the automotive and related industries worldwide. This is probably the best state-of-the-art for small two-stroke engines available today and is a good standard by which other approaches may be compared.

In order to provide a foundation for the evaluation and further development of this unique concept, this report presents an approximate but fairly comprehensive model of the thermodynamic and performance related aspects of the rotary-vee engine. Analyses of the ideal thermodynamic cycle, the in-cylinder flow and heat release process, heat rejection and parasitic losses provide models for the estimation of both indicated and brake performance parameters (however, the model does not treat structural, durability, reliability or cost issues, which must be addressed in the final design/development process). Examples are presented, based primarily on generic input assumptions in lieu of specific technical data, illustrating trends and tradeoffs and defining a level of potential performance which may be attainable after thorough engineering development. Given adequate supporting data, the present analytical model could perform a useful function in that development.

\section{ANALYTICAL RESULTS}

The unique configuration of the rotary-vee engine is illustrated in a general way by the layout cross section drawing reproduced in Figure 5. Clearly, it differs from conventional practice in several significant aspects, to the point where existing analysis programs and data bases do not apply. In this section, simplified, approximate relationships are developed that describe various aspects of this unusual engine. Since these are either highly idealized or dependent on experimental data, which in many cases were not available, the results should be considered illustrative rather than predictive. Although this type of analysis cannot address structural strength and related detail design criteria, it does supply trend and comparison data on output and efficiency. This provides an indication of the performance potential which may be achievable after proper engineering development. In the future, as adequate experimental data become available, the model described in this paper could be further developed to provide a fairly realistic representation of the rotary-vee engine's performance.

\section{Geometry, Basic Dimensions and Piston Motion}

Several geometric and practical constraints apply when the rotary-vee is used as a heat engine. The included angle of the vee has a major effect on the stroke and hence the total cubic displacement within a given package volume; it also affects the load-carrying capacity of the internal structure. Although this angle can theoretically have any value up to nearly $180^{\circ}$, for heat engine purposes the $135^{\circ}$ angle illustrated in Figures 2, 3 and 4 appears to be a good compromise between friction, cubic displacement and strength characteristics. By symmetry, cylinders must appear in multiples of 2 , i.e., one pair associated with each vee-piston. For balance, there must be at least two identical pistons, symmetrically arranged. Thus, the feasible numbers of cylinders for a rotary-vee engine are $4,6,8, \ldots$, etc.. Finally, considerations of volume utilization indicate that a 5- or 6-piston configuration will maximize the ratio of working volume to package volume for a given vee-angle. These constraints virtually dictate the main outlines of the rotary-vee configuration as studied herein: a relatively shallow included vee-angle and 5 or 6 pistons, resulting in 10 or 12 small, under-square cylinders.

Disregarding dimensional details in Figure 5, two of the vee-pistons can be identified: one at top-dead-center (TDC) on the inside of the vee; and the other at bottom-dead-center (BDC) towards the outside or "point" of the vee-shaped structural casing. The two rotating cylinder 
blocks are supported by anti-friction bearings. The wedge-shaped region, running from the point of the vee to the inside corner, houses a mechanical blower which supplies pressurized air to the inlet ports. The blower is formed by placing seal surfaces at the outer and inner envelopes of piston motion within the wedge shaped region. Air is trapped between adjacent pairs of pistons at the wide end, then compressed as the pistons move around towards the narrow end.

The equation for piston motion can be readily derived, using standard kinematics references, e.g. (8), the nomenclature listed at the end of the paper and the geometry illustrated in Figure 6. Clearly the total stroke is

$$
s=2 r_{c} \tan \alpha
$$

and the piston motion (as a function of crank angle) is

$$
r=\frac{s}{2}(1+\cos \psi)
$$

which is similar to the piston motion in a conventional engine, except that higher harmonics are not present. Similarly, the piston velocity is

$$
\dot{r}=-\frac{s}{2} \sin \psi \dot{\psi}
$$

Clearly the total displacement for a 12-cylinder engine is

$$
C I D=3 \pi b^{2} s
$$

The basic dimensions of a typical rotary-vee engine are listed in the following table.

\section{TABLE 1 - TYPICAL ROTARY-VEE DIMENSIONS}

$\begin{array}{lcc}\text { Number of Cylinders, } N_{c}, \ldots \ldots \ldots \ldots \ldots \ldots & 12 \\ \text { Base Circle Diameter, } 2 r_{c}, \mathrm{~cm}(\mathrm{in}), \ldots \ldots \ldots \ldots & 13.3(5.25) \\ \text { Half Vee Angle, } \alpha, \operatorname{deg}, \ldots \ldots \ldots \ldots \ldots \ldots \ldots \ldots & 22.5 \\ \text { Bore, } b, \mathrm{~cm}(\mathrm{in}), \ldots \ldots \ldots \ldots \ldots \ldots \ldots \ldots \ldots \ldots & 3.8(1.5) \\ \text { Stroke } s, \mathrm{~cm}(\mathrm{in}), \ldots \ldots \ldots \ldots \ldots \ldots \ldots \ldots \ldots & 5.524(2.175) \\ \text { Displacement, } C I D, \mathrm{~cm}^{3}\left(\mathrm{in}^{3}\right), \ldots \ldots \ldots \ldots \ldots & 75.58(46.12) \\ \text { Cylinder Compression } / \text { Expansion Ratio, } R_{2}, & 6.7: 1^{*} \\ \text { Blower Compression Ratio, } R_{1}, \ldots \ldots \ldots \ldots \ldots & 1.65: 1^{*} \\ \text { Effective Compression Ratio, } R, \ldots \ldots \ldots \ldots \ldots & 11: 1^{*}\end{array}$

The compression ratios noted above $\left(^{*}\right)$ were inferred from experimental pressure data furnished by the current developer for one test condition. It should be noted that although the overall compression ratio $R$ of this engine is high, the expansion ratio $R_{2}$ (which is most significant for efficiency) is relatively low.

\section{Ideal Thermodynamic Cycle}

As previously noted, the middle portions of the vee-pistons form a positive-displacement blower or air pump if sealing surfaces are provided along the inner and outer envelopes of 
the piston motion near the apex of the vee. The performance of the resulting blower will be estimated in a later section. Here it will suffice to note that with proper attention to the design of the seals, cylinder ports and transfer passages, the blower could become an integral part of the engine's thermodynamic cycle. The resulting blown Otto cycle might be more accurately termed a "compound-compression, single-expansion Otto cycle". This section will investigate the ideal efficiency of this cycle in the rotary-vee engine, using the approach of standard I. C. engine references such as (9).

Figure 7 illustrates the ideal P-V and T-S diagrams for the air-standard Otto cycle with compound compression. The portions bounded by points $1,2,3$ and 4 denote the standard cycle of a conventional engine. In the present case, the main compression stroke $(1-2)$ is preceded by an auxiliary compression $\left(1^{\prime}-1\right)$ in the blower. It is assumed that process $1^{\prime}$ 1 is isentropic and the resulting compressed air is then transferred to the working cylinder without loss. The engine then performs the in-cylinder processes of compression, combustion and expansion in the usual manner. Note, however, that the expansion ratio available for extracting work from this cycle is equal to the in-cylinder compression ratio, $R_{2}$, where

$$
R_{2}=\frac{V_{1}}{V_{2}}=\frac{V_{4}}{V_{3}}
$$

By comparison, the overall compression ratio of the blower and cylinder combination is a higher value, $R$, where

$$
\begin{gathered}
R=R_{1} \times R_{2} \text { and } \\
R_{1}=\frac{V_{1}^{\prime}}{V_{1}}
\end{gathered}
$$

The additional work of compression from $1^{\prime}$ to 1 must come out of the cycle, and on a perunit-mass basis, it is only partially recovered by "working" the cycle at a higher pressure level. The shortfall is best illustrated by the T-S diagram, where it is clear that a constant-pressure process $4^{\prime}-1^{\prime}$ is thermodynamically necessary to bring the blowdown volume $4^{\prime}$ back to the blower intake volume $\mathbf{1}^{\prime}$.

With these ideas in mind, it can be shown that the ideal cycle efficiency, $\eta_{i}$, is given approximately by:

$$
\eta_{i} \approx \eta_{c}\left[1-k\left(R_{1}-1\right)(T R)\right]-\eta_{b}(T R)
$$

where $T R$ is a temperature ratio defined by:

$$
T R=\frac{T_{1}}{T_{3}-T_{2}}
$$

Also, $\eta_{c}$ is the Otto efficiency associated with the in-cylinder cycle 1-2-3-4, $\eta_{b}$ is an Otto efficiency associated with the blower compression ratio, the temperatures are as indicated on the T-S diagram of Figure 7, $\mathrm{k}$ is the isentropic exponent and $R_{1}$ and $R_{2}$ were defined above. Here the terms in the temperature ratio, $T R$, are due to the effect of the idealized mechanical blower. It can be shown that these added terms are non-negative and vanish when $R_{1}=1$, i.e., in the limit of an unblown cycle. 
That is, the efficiency of the rotary-vee cycle is equal to that of the in-cylinder cycle, decreased by terms in the temperature ratio $T R$. For the present study, where nearstoichiometric fuel-air ratios are expected, the above temperature ratio should be small, a fact which justifies the neglect of second-order and higher-order terms in the development of Eq. (6). Nevertheless, the penalty is substantial in comparison with a full-expansion cycle. Hence, there is always a trade off for the power increase (proportional to $R_{1}$ ) which may be obtained by the use of a blower. It must be noted that the efficiency of the rotary-vee engine is set by its in-cylinder compression/expansion ratio $R_{2}$, not by its overall compression ratio, $R$, which may be fairly high.

The ideal specific fuel consumption (SFC) corresponding to Eq. (6) is given by:

$$
\mathrm{SFC}=\frac{2544}{\eta_{i} \times L H V}
$$

where $L H V$ is the lower heating value of the fuel (about $43,200 \mathrm{~kJ} / \mathrm{kg}[18,600 \mathrm{Btu} / \mathrm{lb}]$ for most petroleum-based fuels) and the number 2544 is a conversion factor (Btu/hp-hr).

To complete the idealized analysis, an expression for relative power output is also needed. The power per unit airflow is of course, proportional to $\eta_{i}$. In the rotary-vee, however, the blower is added to an existing piston/cylinder section to increase its output power by increasing its air-handling capacity or apparent displacement. The increase in capacity, relative to the in-cylinder cycle, is given by the blower ratio $R_{1}$; thus, output is proportional to $R_{1} \times \eta_{i}$. Then taking the unblown cycle $\left(R_{1}=1\right)$ as a reference we find the relative power as:

$$
\text { Rel. Pwr. }=R_{1} \times \eta_{i}\left(R_{1}\right) / \eta_{i}(1)
$$

The results of Eqs. (6), (7) and (8) are displayed in Figure 8 for $k=1.4, L H V=43,200 \mathrm{~kJ} / \mathrm{kg}$ $(18,600 \mathrm{Btu} / \mathrm{lb})$ and a range of blower compression ratios chosen to approximately match a limited number of pressure data points furnished by the developer. The results are presented both for the rotary-vee engine and an equivalent, full-expansion engine (where the compression ratio increment $R_{1}$ is applied to the expansion stoke as well as to the compression stroke).

Clearly, both engines gain power as blower compression is added, but the rotary-vee gains the lesser amount. In terms of efficiency and SFC, the rotary-vee performance deteriorates with increasing $R_{1}$ while the full-expansion engine continues to improve. In fact, if $R_{1}$ were allowed to increase to a value in the neighborhood of $R_{2}$, the rotary-vee output curve would roll over and approach zero. In this limiting case (not shown in Figure 8), the in-cylinder work (area 1-2-3-4 in Figure 7) would be exactly balanced by the blower compression work $1^{\prime}-1$ and the machine would produce no net power at all. It might serve, however, as an effective gas generator under these conditions.

Actually, the values shown in Figure 8 are extremely optimistic for both engines. The values should realistically be degraded by factors approaching 2 to account for various losses and non-ideal processes. At this point it is difficult to argue that real-world effects apply differently to the two engines. Hence, the percentage penalties for the rotary-vee cycle as compared to the full-expansion cycle may be representative, if not exact. The short expansion stroke of the rotary-vee represents a major thermodynamic loss. In the limit, it is a better gas-generator than a shaft-power engine. For best efficiency, the use of a compounding turbine should be considered. This would enable the engine to recover some portion of the relatively large blow-down energy (process $4-4^{\prime}$ ) shown in Figure 7. Alternatively, the presently-unused return side of the blower might be used as a positive displacement expander. Then, assuming 
solutions for various thermal and mechanical design problems, the full-expansion SFC and power outputs might still be obtained from an attractively sized package.

\section{Combustion Chamber Heat-Release Analysis}

In order to more realistically assess the chamber pressure profile and related internal parameters in the rotary-vee engine during a cycle, the chamber was modeled as an open thermodynamic system. This approach has been used successfully by Gatowski et al. (10), for a conventional spark ignition engine, diesel engines and a direct-injection stratified charge engine; by Norman (11) for a carbureted internal combustion engine; and by Roberts (12) for a direct-injection stratified charge engine. During intake, exhaust and compression, the chamber contents are modeled as a uniform mixture of gases at a single pressure and temperature. During the combustion and expansion processes, the chamber contents are divided into a burnt and an unburnt zone. Mass flows across the system boundaries include intake and exhaust flows. Energy crossing the boundaries (aside from that associated with mass flows) include heat transfer to the cylinder walls, the piston top and the head, and work on the piston. Following are brief descriptions of major parts of the model, discussion of the model's validity and a summary of results.

Intake and exhaust flows are modeled as quasi-steady compressible flows. Because no flow rate data were available, intake port and exhaust port discharge coefficients were set equal to 1.0. Intake manifold pressure and exhaust manifold pressure were held constant through the cycle and were calculated as described in the blower analysis section of this report. During the scavenging process, complete mixing was assumed so that the exhausted gas has the same instantaneous composition as the chamber gas.

Intake and exhaust port open areas and timings for one version of the engine were provided by the developer (Figure 9). The port open area profiles are not symmetrical about BDC because of the piston crown shape and the rotating piston motion of the rotary-vee pistons. The piston crowns are slanted, such that as the pistons rotate in their sleeves, the exhaust port is opened before the intake port opens and also closes before the intake port closes. Because of this port timing feature, the rotary-vee engine scavenging efficiency is expected to be greater than that of a conventional, loop-scavenged two-stroke engine with symmetrical port timings, and close to the scavenging efficiency of an opposed piston two-stroke diesel engine (which also employs unsymmetrical port timing). The scavenging efficiency of the opposed piston diesel engine is very close to that of a hypothetical engine in which perfect mixing is assumed during intake and exhaust (9).

As noted earlier, during combustion the chamber was divided into a burnt and an unburnt zone. As combustion proceeds, mass flows from the unburnt zone to the burnt zone according to

$$
x_{b}=1-\exp \left[-a\left(\frac{\theta-\theta_{o}}{\Delta \theta}\right)^{m+1}\right]
$$

where $x_{b}$ is the mass fraction of burnt products in the chamber, $\theta$ is the crank angle, $\theta_{o}$ is the crank angle at which the spark fires, $\Delta \theta$ is the expected duration (in crank angle degrees) of combustion and $a$ and $m$ are empirical constants.

It will be noted that Eq. (9) is a function of crank angle and does not depend explicitly on time or engine speed. This type of expression has been found to be appropriate for a wide range of I. C. engine circumstances because, to a first order of approximation, the flame speed 
of the combustion front is proportional to engine speed. Thus, both the flame speed and the piston speed increase proportionally to engine speed and combustion events correlate directly to crank angle.

The particular expression used here was originally proposed by Wiebe (13) and has been used by Norman (11) for a carbureted internal combustion engine. Ideally, the constants a and $m$, the spark advance, $\theta_{o}$, and the combustion interval, $\Delta \theta$, would be adjusted such that predicted chamber pressure would match experimental values. In this case, no experimental chamber pressure data were available, so the constants were perturbed until the resulting pressure profile shape was judged to be representative of good practice for small gasoline engines. The resulting constants were: spark advance $\theta_{0}=15^{\circ}$ BTDC; combustion interval $\Delta \theta=45^{\circ} ; a=2.0$ and $m=1.0$. The same values were used for all values of engine speed in this section. Thus, except for the relatively small effects of heat losses, the work output per cycle at all speeds is proportional to the mass of air/fuel mixture trapped in the cylinder.

By the above procedure, the rotary-vee engine is credited with a level of combustion performance which, in other I. C. engines, has been obtained only after substantial in-cylinder development efforts were accomplished. This, combined with the previous assumption of complete mixing during the scavenging process, may result in significant over-prediction of the capabilities of the rotary-vee engine. It does, however, provide an indication of the potential that might be expected with proper engineering development.

Convective heat transfer to the cylinder walls, cylinder head and piston top was calculated using the expression proposed by Woschni (14):

$$
N u=\beta \operatorname{Re}^{g} \operatorname{Pr}^{h}
$$

where $N u$ is Nusselt number, $R e$ is Reynolds number, $\operatorname{Pr}$ is Prandtl number and $\beta, g$ and $h$ are empirical constants. Again, because no cooling flow rate and temperature data were available, the constants were set equal to the values used in a previous analysis of a carbureted internal combustion engine: $\beta=0.037, g=0.8$ and $h=1.0$. Average gas velocity during intake, exhaust and compression was set equal to the piston velocity. During combustion, an auxiliary term was added to the average gas velocity to represent the turbulent motion of the burning gases.

The above equations were used, along with the conservation of mass and energy, the ideal gas relation and expressions for chamber volume and its derivative to form a system of non-linear first order differential equations. These equations were integrated numerically. Two expressions for chamber volume and volume derivative were used: one for the rotary-vee engine employing simple harmonic motion for the piston and the other for a crank piston engine which includes higher harmonics. The volume relations are given by Eqs. (2) and (17) to (20). As will be seen later, the difference in the piston motions of a rotary-vee and crank piston engine results in small differences in the peak chamber pressures and crank angle for peak chamber pressure for the two engines. Crank piston peak chamber pressures are lower and occur later in the combustion process than for the rotary-vee engine when the spark timing for both is $20^{\circ} \mathrm{BTDC}$. Initial chamber temperature and pressure (at the time the intake port opens) were chosen and the equations were integrated through a cycle. Final temperature, pressure and mass were compared to initial values, and if any one of the differences was too great, new initial values were estimated and integration was repeated.

As previously noted, the model's constants could not be calibrated, because of a lack of experimental data. Therefore, the model should not be expected to rigorously predict such 
quantities as IMEP or maximum chamber pressure. The model's results are useful, however, both because they show the effect that changing such operating conditions as manifold pressures and engine speed have on the rotary-vee's performance and because they indicate a reasonable level of the engine's potential capabilities.

Figure 10 summarizes the results of the combustion chamber gas heat-release analysis. It shows pressure profiles of the rotary-vee engine at various engine speeds and intake manifold pressures. The peak work output per cycle and the greatest maximum chamber pressure occur when the rotary-vee is run at $5200 \mathrm{rpm}$. Because of the blower/cylinder airflow matching considerations discussed in the next section, the inlet manifold pressure (MP) rises with engine speed until reaching a value of 2.0 atm at $5200 \mathrm{rpm}$. The work output per cycle, as previously mentioned, is proportional to the mass of charge trapped in the cylinder, which, in turn, increases almost linearly with MP. Thus, the rotary-vee should exhibit indicated mean effective pressure and torque characteristics which vary almost linearly with engine speed, up to about $5200 \mathrm{rpm}$ for the port timings of Figure 9 . Above $5200 \mathrm{rpm}$, the residual burnt gas fraction increases with engine speed, resulting in less fuel mass trapped per cycle and a consequent reduction in peak pressure during the cycle.

At low speeds the intake manifold pressure is low and heat transfer to the cylinder walls and head represents an appreciable fraction of the input fuel energy, as shown in Figure 11. At higher speeds the cooling losses become less significant on a per cycle basis because the time available for heat transfer per cycle decreases while the fuel burnt per cycle increases.

Figure 12 shows indicated specific fuel consumption (ISFC) for various engine speeds. ISFC was calculated using both the fuel mass trapped in the chamber at TDC (simulating direct-cylinder fuel-injection) and also the fuel mass that passes through the intake port (simulating the carbureted case). As with other piston-ported two-stroke engines, there is a substantial amount of blowthrough (of fresh charge through the exhaust port) for the rotaryvee at low speeds. In fact, the blowthrough for the rotary-vee engine will be greater than for other two-stroke engines because the fresh charge comes into the cylinder at an elevated pressure from the blower (between the cylinder blocks). It is evident that, at all speeds, a direct-injection scheme would benefit the rotary-vee engine greatly.

It has been suggested that at high speeds the rotary-vee engine can be run with a lean fuel/air mixture. The rotary-vee spark plugs are offset to the outside of the cylinder heads, and, according to one theory, the fuel charge may become stratified near the spark plug at high speeds (4). This could be caused by the centrifugal force on fuel droplets from the spinning cylinder blocks. This effect is considered speculative, however, since it has not been shown experimentally. In any case, stratified charge combustion is a highly complex subject which cannot be adequately simulated in the present type of analysis.

\section{Blower and Cooling Power Losses}

As previously described, the annular space at the apex section of the rotary-vee engine (between the two cylinder blocks, Figure 5) can be used as a compressor for the mixture provided to the cylinders. The outside and inside envelopes of the pistons' motion are sealed so that air and fuel (from the carburetor) are trapped between successive pistons. Fuel/air mixture flows into the blower where the volume between successive pistons is a maximum, flows out of the blower where the volume is a minimum and then flows into the cylinders. The blower exit pressure is fixed by the constraint that air flow demanded by the cylinders must match air flow provided by the blower. 
Using the thermodynamic model of the chamber gases, air mass flow rate demanded by the 12 cylinders was computed and plotted for intake manifold pressures (blower exit pressures) of $1.1 \mathrm{~atm}, 1.33 \mathrm{~atm}, 1.67 \mathrm{~atm}$ and $2.0 \mathrm{~atm}$ and engine speeds ranging from $500 \mathrm{rpm}$ to $6500 \mathrm{rpm}$. These curves are nearly horizontal and are shown in Figure 13. Note that these curves reflect the port timings and areas that were used in this study (Figure 9) and that the engine could be readily tuned to higher or lower speeds with different port characteristics. Next, the maximum volume of air trapped between the pistons, $V_{t}$, was calculated and the air mass flow rate delivered by the blower was calculated according to

$$
\dot{m}_{a i r}=\frac{V_{t} \times 6 \times R P M / 60}{v_{a i r}}
$$

where $R P M$ is engine speed, $v_{a i r}$ is the specific volume of the air at the blower inlet and the 6 appears because there are passages between 6 pistons. This theoretical blower capacity is also plotted in Figure 13. At $1800 \mathrm{rpm}$, a measured boost pressure of 3 psi (i.e., blower exit pressure is $1.204 \mathrm{~atm}$ ) has been reported. The chamber gas thermodynamic model was run for these conditions and the cylinder air demand was found to lie very close to the theoretical blower capacity line.

In order that air demanded by the cylinders equals air supplied by the blower, the rotaryvee operates along the theoretical blower capacity line in Figure 13 up until about 5200 rpm. At 5200 rpm the blower exit pressure reaches the maximum blower exit pressure reported by the developer, 2 atm. Above $5200 \mathrm{rpm}$ the blower is assumed to maintain a constant exit pressure of 2 atm.

The work that the blower requires to compress air from atmospheric pressure to the blower exit pressure was calculated assuming that only air is compressed, that the air behaves as an ideal gas and that the compressor has an efficiency of $80 \%$.

Because work is required to spin the barrels (against air resistance), there will also be a cooling and windage power loss. It was reported that, at $6000 \mathrm{rpm}$, with the pistons removed, between $1.5 \mathrm{~kW}$ and $3 \mathrm{~kW}$ ( $2 \mathrm{hp}$ and $4 \mathrm{hp}$ ) was required to motor the rotary-vee cylinder blocks. Assuming the higher value, the cooling power (in $\mathrm{kW}$ ) at other engine speeds is conservatively estimated by the fan law:

$$
\text { Cooling Power }=3 \mathrm{~kW}\left(\frac{R P M}{6000}\right)^{3}
$$

Thus, cooling power is not a large penalty for the rotary-vee at speeds below $6000 \mathrm{rpm}$. The use of ceramic heat barrier coatings in the combustion chamber surfaces could reduce this already low value by a factor of approximately 2 via proportional reductions of the cylinder heat rejection shown in Figure 11. Thus, based on the reported measurement of cooling power required, a savings of $0.75-1.5 \mathrm{~kW}(1-2 \mathrm{Hp})$ at $6000 \mathrm{rpm}$ could be expected from the use of such adiabatic coatings.

\section{Output Torque and Friction}

A simple kinematic analysis was performed for the rotary-vee engine to determine indicated torque, friction torque and brake torque. The geometry used in this analysis is shown in Figure 14. The friction torque has three primary components: (1) that due to the sliding of the pistons; (2) that due to the rotation of the pistons; and (3) that due to the sliding of the rings. Friction associated with the anti-friction cylinder block bearings was neglected. 
The forces the rings exert on the sleeve are shown in Figure 15. $P_{o}$ is the pressure equivalent of the ring pre-tension, which is independent of chamber pressure. In addition to its pre-tension, the first ring also "sees" the full chamber pressure, $P(\psi)$, and exerts an integrated normal load on the cylinder wall as shown. The instantaneous pressure between the rings (exerted on the second ring) was estimated to be half the instantaneous chamber pressure.

Indicated torque, $\tau_{i}$, friction torque due to the piston sliding, $\tau_{f}$, friction torque due to the piston rotating, $\tau_{t}$, and friction torque due to the rings sliding, $\tau_{r}$, were found to be

$$
\begin{gathered}
\tau_{i}=P(\psi) A \tan \alpha r_{c} \sin \psi \\
\tau_{f}=-\mu_{p}\left|P(\psi) A \tan \alpha-m_{p} \omega^{2} r_{c} \cos \psi\right| \times \\
r_{c} \tan \alpha \sin \psi \sigma(\dot{r}) \\
\tau_{t}=-\mu_{p} r_{p}\left(F_{c}^{2}+F_{s}^{2}+2 F_{c} F_{s} \cos \psi\right)^{1 / 2} \\
\tau_{r}=-3 \pi \mu_{r} r_{p} h_{r}\left[P_{o}+P(\psi)\right] r_{c} \sin \psi \tan \alpha \sigma(\dot{r})
\end{gathered}
$$

where

$$
\sigma(\dot{r})=\frac{\dot{r}}{|\dot{r}|}
$$

The variables used in Eqs. (13) to (16) are defined in the Nomenclature. Note that Eq. (15) describes a component of the forces on the piston and a friction torque that are unique to the rotary-vee engine, namely the centrifugal force and the tangential friction torque due to the spinning of the barrel. At high engine speeds, the centrifugal force is large and the tangential torque exceeds the torque due to the sliding of the rings. No analog to Eq. (15) exists for a conventional crank piston engine.

The values chosen for the coefficients of sliding friction for the piston and rings were $\mu_{p}=0.01$ and $\mu_{r}=0.02$. The coefficient of sliding friction for the rings was chosen as larger because oil is squeezed from the ring/cylinder interface as the rings slide over the sleeve. The pre-tension of the rings was arbitrarily chosen to be equivalent to 23.8 atm (350 psi), to preclude leakage during the compression process.

\section{Torque and Friction of a Crank Piston Engine}

In this section, the torque and friction relationships applicable to a crank piston engine are shown for comparison with Eqs. (13) to (16) above. Figure 16 shows the geometry used in the simple kinematic analysis of a conventional, two-stroke, crank piston engine and the forces on it. The force terms are nearly the same as those for the rotary-vee engine, with two exceptions: (1) there is no direct centrifugal force term at all in the crank piston engine; and (2) since the piston motion is not simple harmonic, the linear inertia term corresponding to Eq. (14) must be modified to account for higher harmonics. Using the customary harmonic expansion (9), it can be shown that

$$
r(\psi)=r_{c}\left[(1+\cos \psi)+a_{2}(\cos 2 \psi-1)+\right.
$$




$$
\left.a_{4}(\cos 4 \psi-1)+\cdots\right]
$$

and

$$
\ddot{r}(\psi)=-\omega^{2} r_{c}\left(\cos \psi+4 a_{2} \cos 2 \psi+16 a_{4} \cos 4 \psi+\cdots\right)
$$

where

$$
\begin{gathered}
a_{2}=\frac{l}{r_{c}}\left[\frac{1}{4}\left(\frac{r_{c}}{l}\right)^{2}+\frac{1}{16}\left(\frac{r_{c}}{l}\right)^{4}+\frac{15}{512}\left(\frac{r_{c}}{l}\right)^{6}+\cdots\right] \\
a_{4}=-\frac{l}{r_{c}}\left[\frac{1}{64}\left(\frac{r_{c}}{l}\right)^{4}+\frac{3}{256}\left(\frac{r_{c}}{l}\right)^{6}+\cdots\right]
\end{gathered}
$$

and so forth. Since $r_{c} / l$ is typically on the order of $1 / 3$, it can be seen that terms of the sixth order and higher are negligible. Keeping in mind the above definitions, the indicated torque, friction torque due to piston sliding and friction torque due to the rings are given by

$$
\begin{gathered}
\tau_{i}=\left[P(\psi) A-m_{p}^{\prime} \ddot{r}(\psi)\right] r_{c} \sin \psi \\
\tau_{f}=-\mu_{p}\left|\tau_{i}\right| \tan \alpha \sigma(\dot{r}) \\
\tau_{r}=-3 \pi \mu_{r} r_{p} r_{c}^{\prime} h_{r}\left[P_{o}+P(\psi)\right] \sin \psi \sigma(\dot{r})
\end{gathered}
$$

Here, $r_{c}$ is half the stroke and $m_{p}^{\prime}$ is the piston mass. As previously noted, the crank piston engine has no friction component that corresponds to the rotary-vee centrifugal/rotational term expressed in Eq. (15). Consistent with the rotary-vee analysis, the losses of the antifriction main, rod and wrist-pin bearings of the crank piston engine were assumed to be negligible.

\section{DISCUSSION}

Eqs. (13) to (23) were programmed and evaluated, using chamber pressure profiles calculated by the thermodynamic cycle analysis of the chamber gas. Indicated torque, friction torque and output torque were calculated at each crank angle and averaged over a full revolution. From these averages, indicated and brake horsepower and specific fuel consumption were calculated.

Output torque, power etc. were calculated for both the rotary-vee and an equivalent crank piston two-stroke engine. The equivalent crank piston engine was given the same bore and stroke as the rotary-vee, as well as the same ring tensions and friction coefficients. In both cases, the friction contributions due to various anti-friction bearings were neglected. Recall that the pressure profiles used in the two engines differ somewhat, because the piston motion in the rotary-vee engine is simple harmonic, while that of the crank piston engine was not. Both engines were assumed to be supercharged to the same intake manifold pressure.

Figures $17 \mathrm{a}$ and $\mathrm{b}$ show the instantaneous output torque for the rotary-vee and crank piston engines versus crank angle for engine speeds of $1250 \mathrm{rpm}$ and $4200 \mathrm{rpm}$, respectively. Figure 18 is a plot of average indicated torque for various engine speeds for the rotary-vee and crank piston engines. Figure 19 is a plot of output horsepower for the same engines at various 
engine speeds. Note that cooling and compressor power losses are not included in Figures 17, 18 and 19.

Indicated torque and horsepower are different for the two engines, for two reasons. First, as described earlier, the pressure profiles for the two engines (during a cycle) differ because the rotary-vee engine chamber volume changes according to simple harmonic motion, while the volume expression for the crank piston engine involves higher harmonics. Second, the spark timings for the engines were optimized only to within about $\pm 2^{\circ}$; it is expected that the indicated torques of the two engines will be closer for optimized spark timings.

The most significant result from the comparison of the rotary-vee and crank piston engines is shown in Figure 19. At high speeds, the friction losses for the rotary-vee engine are substantially greater than those for the crank piston engine. This is due to the friction effects that arise from the spinning of the rotary-vee barrels. Recall that the rotary-vee centrifugal friction forces have no counterpart in the crank piston engine.

Figure 20 shows indicated power, brake power and parasitic losses (including friction losses, compressor losses and cooling losses) and specific fuel consumption for the rotary-vee engine for various engine speeds, but it must be noted that the magnitude of the indicated power may be inaccurate, since there was no chamber pressure data for calibration of the chamber gas thermodynamic model. However, this figure does show that parasitic losses are a significant fraction of output power at high speeds. As engine speed is increased from $4200 \mathrm{rpm}$ to $6000 \mathrm{rpm}$, the sum of the blower and cooling losses nearly doubles. Friction losses more than double from $4200 \mathrm{rpm}$ to $6000 \mathrm{rpm}$. Despite increasing friction losses, BSFC decreases with speed for the carbureted rotary-vee engine, because the mass of fuel blown through the engine decreases with increasing engine speed. For a direct-cylinder fuel-injection engine, the BSFC increases slowly with engine speed. Again, it is noted that significant advances in fuel economy could be made for the rotary-vee engine if a direct-injection scheme were employed. This is the largest potential improvement for the rotary-vee engine that was identified in this study.

In summary, the rotary-vee may offer a viable but not exceptional level of performance. As previously demonstrated, the application of advanced technologies to the rotary-vee could significantly improve its performance. The addition of a direct-cylinder injection scheme offers the greatest potential improvement in performance, while using a full expansion cycle would also result in significant gains. It was shown that friction losses are a greater problem for the rotary-vee than for an equivalent crank piston engine. These friction losses could be decreased by the use of a lighter piston, improved oil or improved oiling techniques. A small improvement in SFC could be accomplished through the use of adiabatic coatings on the cylinder walls, thus reducing the power required for cooling the cylinders. The potential improvements offered by the application of advanced technology to the rotary-vee engine are shown in Table 2 


\section{TABLE 2 - POTENTIAL IMPROVEMENTS DUE TO NEW TECHNOLOGIES}

\author{
Technology \\ Use of Direct-Cylinder \\ Fuel Injection \\ Use of a Full-Expan- \\ sion Cycle \\ 50\% Reduction in Friction \\ via Lighter Piston, \\ Improved Oiling \\ Adiabatic Combustion \\ Chamber Coating \\ (50\% reduced cooling \\ power loss)
}

\section{Benefit}

$30 \%$ to $120 \%$

improved SFC

$15 \%$ to $25 \%$

improved SFC

and power

$8 \%$ improved SFC

and power

$1 \%$ to $2 \%$

improved SFC

and power

\section{CONCLUSIONS}

An analysis of the rotary-vee engine has been conducted to support an apparent industrial interest. The supporting calculations were presented in the preceding sections of this report and the major findings and conclusions are reviewed below.

a. Briefly, the rotary-vee is an unusual two-stroke internal-combustion engine which incorporates many small cylinders in a pair of rotating cylinder blocks. As in the rotary-radial engines developed and used around 1915, piston reciprocation occurs as a relative motion between rotating parts. Its mechanical simplicity and inherent balance make it a potentially low cost and light weight candidate for appropriate applications. The current design also includes a built-in mechanical blower and rotary control of inlet and exhaust ports. It operates on the "blown" or mechanically supercharged Otto cycle which was widely used in World War II aircraft reciprocating engines.

b. An immediate finding is that the engineering status of the rotary-vee engine is less mature than optimistic media reports might suggest. It appears that no overall cycle and internalprocess model for the rotary-vee existed, prior to the new analysis prepared for this study. Experimental and/or computational studies on several basic engineering issues, such as blower performance, apparently have not yet been accomplished. As far as could be determined in this study, no rotary-vee engine-dynamometer testing has been reported during the past 5 years.

c. For lack of adequate engineering information, a new analytical computer program was developed by NASA. The program includes first order treatments of the thermodynamic cycle, internal flow and combustion events, friction and parasitic losses, but does not address structural strength or other detail design criteria. The results indicate that the rotary-vee is thermodynamically feasible, but no clear advantage over a conventional, but state-of-the-art 
alternative, such as the Orbital two-stroke, fuel-injected engine, could be found. By comparison, the rotary-vee appears to have a higher power density but a lower efficiency. These attributes are typical of the mechanically-blown cycle (not to be confused with a modern turbocharged cycle) where a power boost is normally accompanied by decreased efficiency.

d. New technology is, of course, an avenue for improving the efficiency of the rotary-vee. Currently, the use of direct-cylinder fuel-injection, as in the Orbital engine, appears to be the most important new technology issue for small, low-cost, two-stroke engines. The Orbital technology is being actively licensed in the automotive and outboard marine engine industries at present and is presumably available to other industries on a royalty basis. The reported efficiency and emissions benefits for this technology are significant and it is difficult to contemplate developing an all-new, two-stroke engine without considering it. The present simulations show the expected, factor-of- 2 type improvements in SFC when direct-cylinder fuel-injection is assumed for the rotary-vee. In principle, the Orbital or equivalent fuel-injection technology could be applied to the rotary-vee. However, this application of direct-injection technology may not be advantageous because of the greater number of cylinders in the rotary-vee (12 vs. 2 or 3 ) and the rotating cylinder block configuration. These same factors make a diesel version unlikely.

e. The analysis also shows significant (15\% to $25 \%)$ potential improvements in both power and efficiency by the use of either a full-expansion power cycle, a compounding turbine or other exhaust heat recovery device. It should be noted, however, that conventional engines inherently use the full-expansion cycle, while its application to the rotary-vee may not be straightforward. Similarly, turbocompounding is an existing technology which can be applied to a conventional engine whenever needed. Its use in the rotary-vee, however, would be complicated by the whirling exhaust ports.

f. It was shown that the friction losses of the rotary-vee are appreciably greater than those of a comparable crank piston engine. Although the friction losses associated with gas-pressure loads are similar, the rotary-vee has an additional component, not present in a conventional engine. This is due to the steady centrifugal load on the orbiting pistons, acting against the relative piston/cylinder rotating motion. This component of friction loss is proportional to the cube of engine speed, and though insignificant at low speeds, it becomes important above $4000 \mathrm{rpm}$. While the estimated friction losses of the rotary-vee were not prohibitive, this is yet another area where no advantage could be found.

The centrifugal friction losses could be reduced by reducing the weight of the pistons, for instance by making them of a composite material with an advanced ceramic wear coating. The possible savings are rather limited ( $8 \%$ or less) since the present aluminum-alloy piston is very light. And in this case, the primary effect would be to somewhat reduce a loss that is not present at all in a conventional engine. Finally, the use of ultra-light components to reduce friction is not limited to the rotary-vee but should benefit all $\mathrm{I}$. C. engines.

g. Finally, advanced ceramic heat barrier coatings could be applied to reduce the cooling power losses of the rotary-vee. But, as is the case with other I. C. engines, this offers little benefit unless accompanied by turbocompounding or the equivalent. The rotary-vee already has a low cooling power loss and heat rejection rate, and there would be minimum further benefit ( $1 \%$ to $2 \%$ ) from ceramic heat barrier technology alone.

h. The numerous cylinders and rotating cylinder blocks inherent in the rotary-vee design represent a difficult environment for research instrumentation. This configuration cannot be represented with a stationary, one cylinder research rig. In modern I. C. engine development, 
literally dozens of high response, simultaneous measurements of in-cylinder pressure, temperature, flow and related parameters are required on a continuing basis. For the rotary-vee, this requires the use of auxiliary systems to translate the data from the rotating environment to the laboratory environment. Although by no means impossible, this adds a substantial degree of complexity and cost and more opportunities for error to the research and development operation. As a practical matter, therefore, the further development of the rotary-vee engine may prove to be unexpectedly difficult.

In conclusion, the present study indicates that the rotary-vee is thermodynamically feasible, but has no clear advantage over conventional, state-of-the-art, two-stroke internal combustion engines. In comparison with a conventional engine, the rotary-vee may have an advantage in terms of specific power, but it has a less efficient theoretical cycle and higher friction losses. It does have several noteworthy features such as a built-in mechanical blower and rotary control of the inlet and exhaust ports. These features may allow it to be tuned effectively for specific applications, particularly where size and weight are more important than fuel consumption. 


\section{NOMENCLATURE}

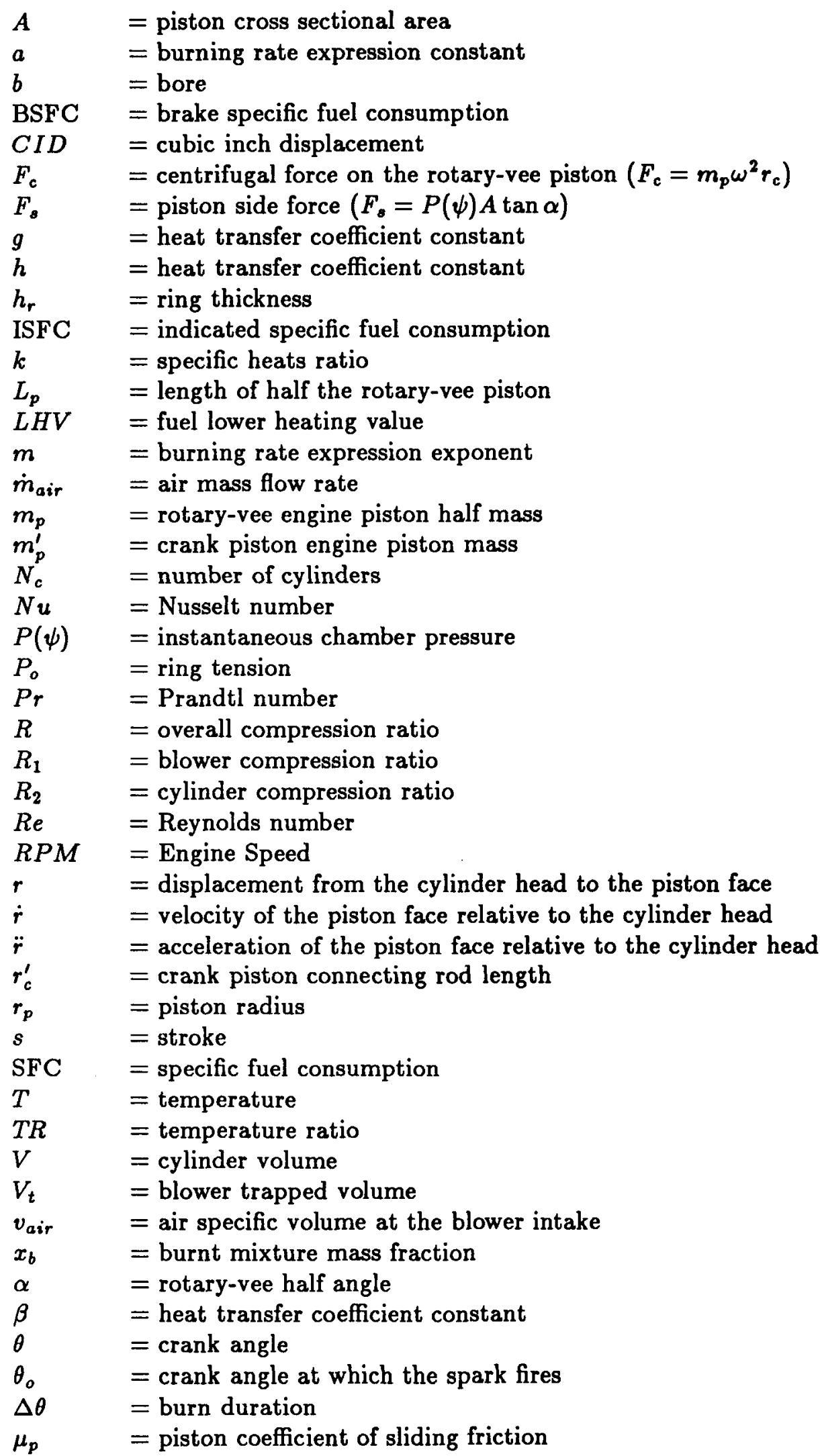


$\mu_{r} \quad=$ rings coefficient of sliding friction

$\eta_{i} \quad=$ ideal cycle efficiency

$\eta_{c} \quad=$ Otto efficiency

$\psi \quad=$ rotation angle of the barrel (crank angle)

$\omega \quad=$ barrel or crank shaft angular velocity

\section{REFERENCES}

(1) E.F. Lindsley, "Rotary V", Popular Science, August, 1974.

(2) B. Tripolsky, "Bricklin Comes Back With a Rotary Engine", Mechanix Illustrated, January, 1977.

(3) D. Calvin, "Rotary Vee Engine", Sport Aviation, February, 1986.

(4) Anon., "Oklahoma Inventor Thinks Rotary-V Worth a Look", Ward's Engine Update, January, 1989.

(5) Anon., "Bricklin/Turner test results", Doug Roe Engineering, Tempe, Arizona, 1977.

(6) Anon., "to Stroke... Or Not Two Stroke", Automotive Industries, May, 1988.

(7) Anon., “Ward's Wrapup”, Ward's Auto World, August, 1988.

(8) Doughty, S. "Mechanics of Machines", Chapter 7, Wiley, 1988.

(9) Taylor, C.F., "The Internal Combustion Engine in Theory and Practice", Vol. 1, Chapters 2 and 7 and Vol. 2, Chapter 8, The MIT Press, 1985.

(10) Gatowski, J.A., et al., "Heat Release Analysis of Engine Pressure Data", SAE 841359, 1984.

(11) Norman, T.J., "A Performance Model of a Spark Ignition Wankel Engine: Including the Effects of Crevice Volumes, Leakage and Heat Transfer", Master's Thesis, Massachusetts Institute of Technology, June, 1983.

(12) Roberts, J.M., "Heat Release Estimation and Prediction of Wankel Stratified-Charge Combustion Engine", Master's Thesis, Massachusetts Institute of Technology, September, 1985.

(13) Wiebe, J.J., "Das Wiebe - Brenngesitz ein Fortschnitt in der Kreigspozesse von Verbrennungsmotoren", Trans. by Prof. A. Joute and T.H. Dressen, Kraftadrzeugtechnik, Vol. 9 (1960).

(14) Woschni, G., "A Universally Applicable Equation for the Instantaneous Heat Transfer Coefficient in the Internal Combustion Engine", SAE 670931, 1967. 


\section{ORIGINAL PAGE \\ BLACK AND WHITE PHOTOGRAPH}

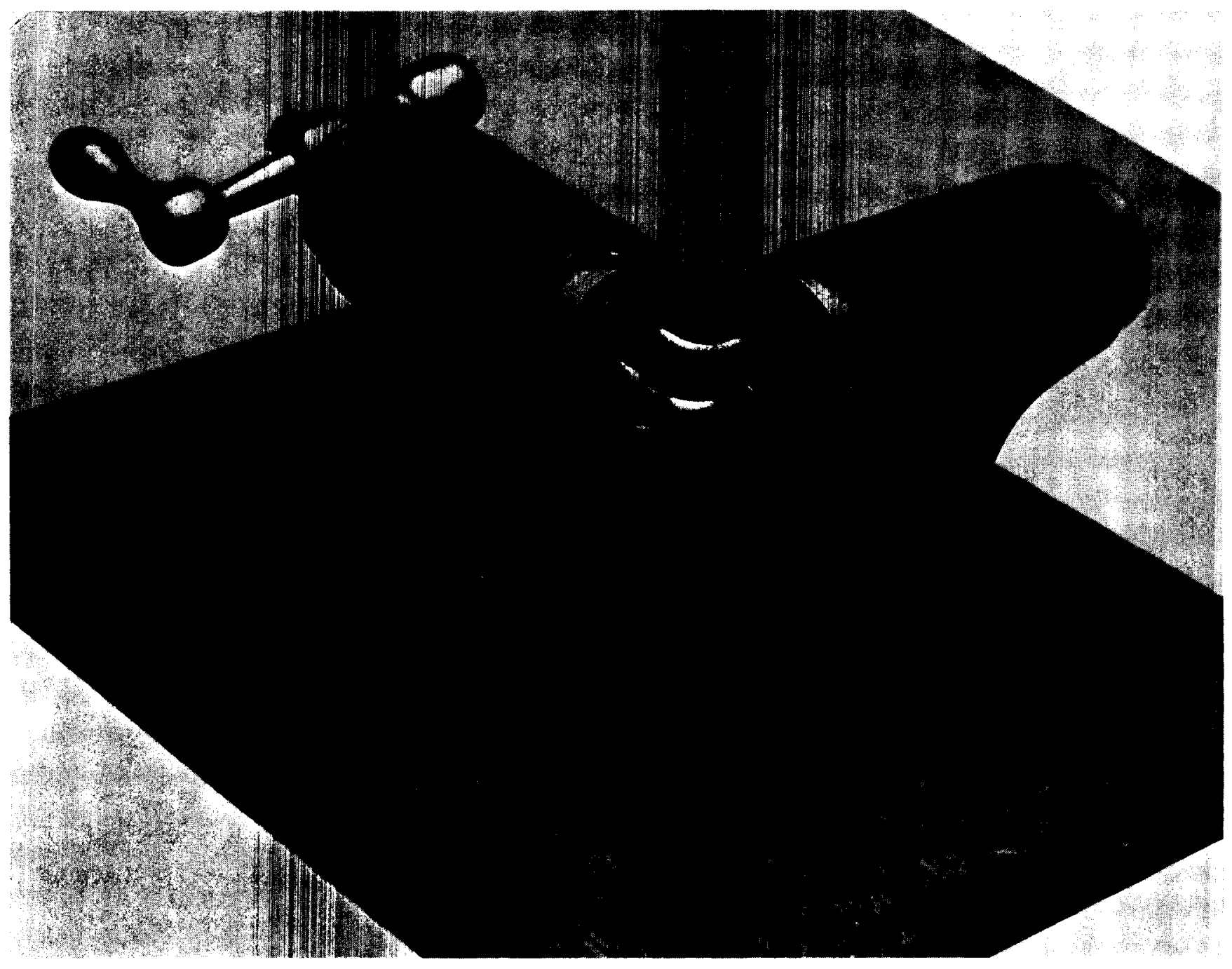

Figure 1 -- Model of a Four Piston Rotary-Vee Mechanism (Two Pistons Removed for Clarity) 


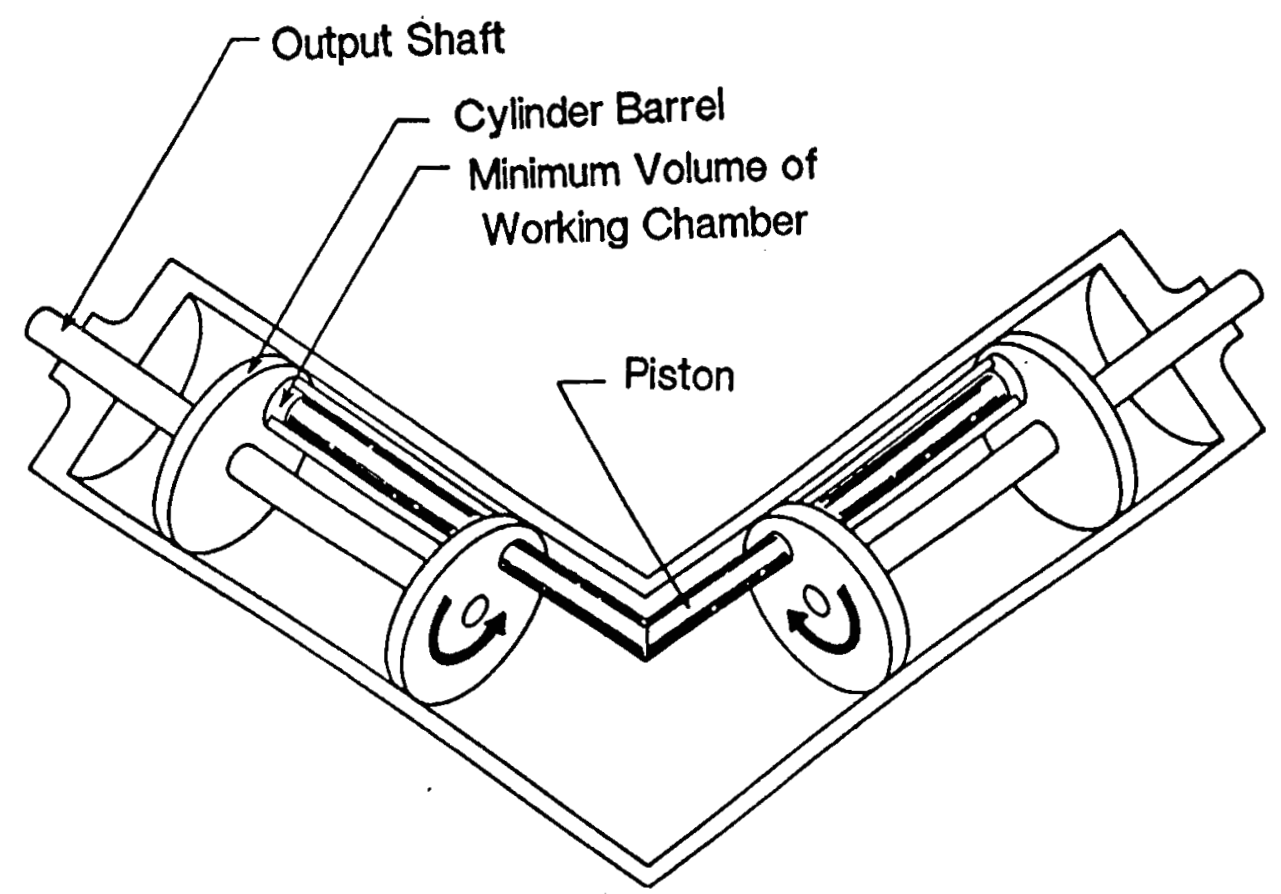

Figure 2 -- The Rotary-Vee Engine With the Piston at TDC

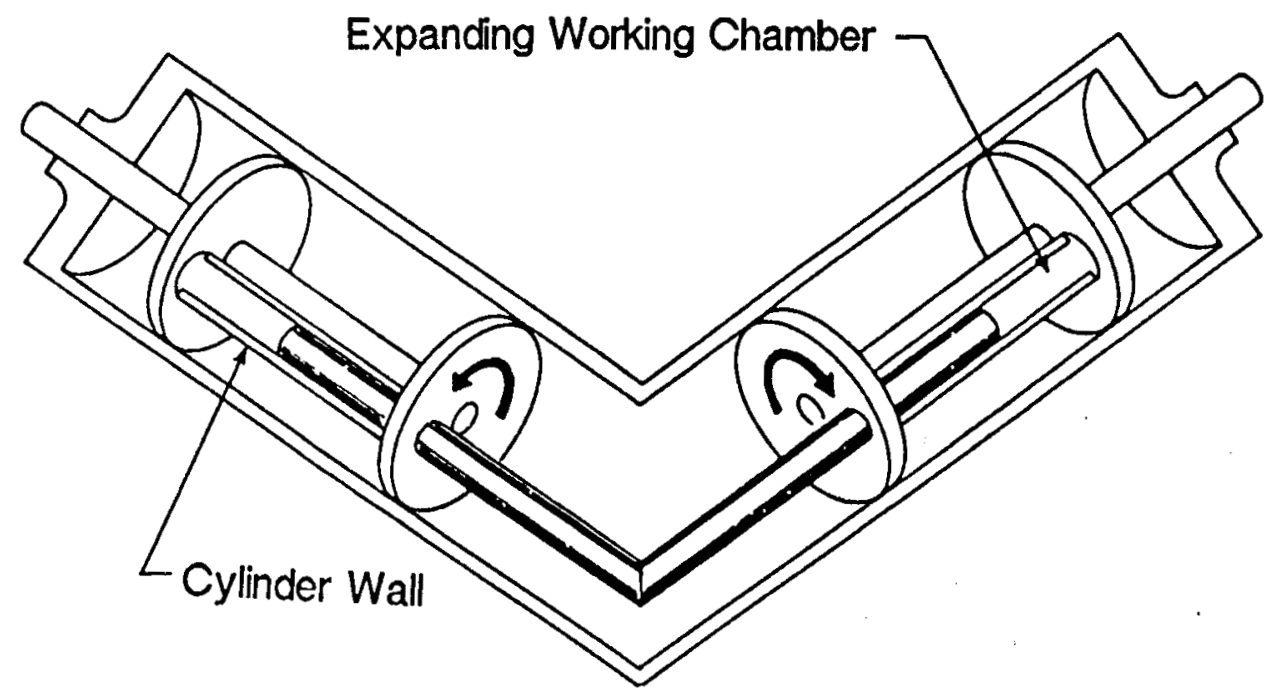

Figure 3 -- The Rotary-Vee Engine After the Piston Has Rotated 120 Degrees 


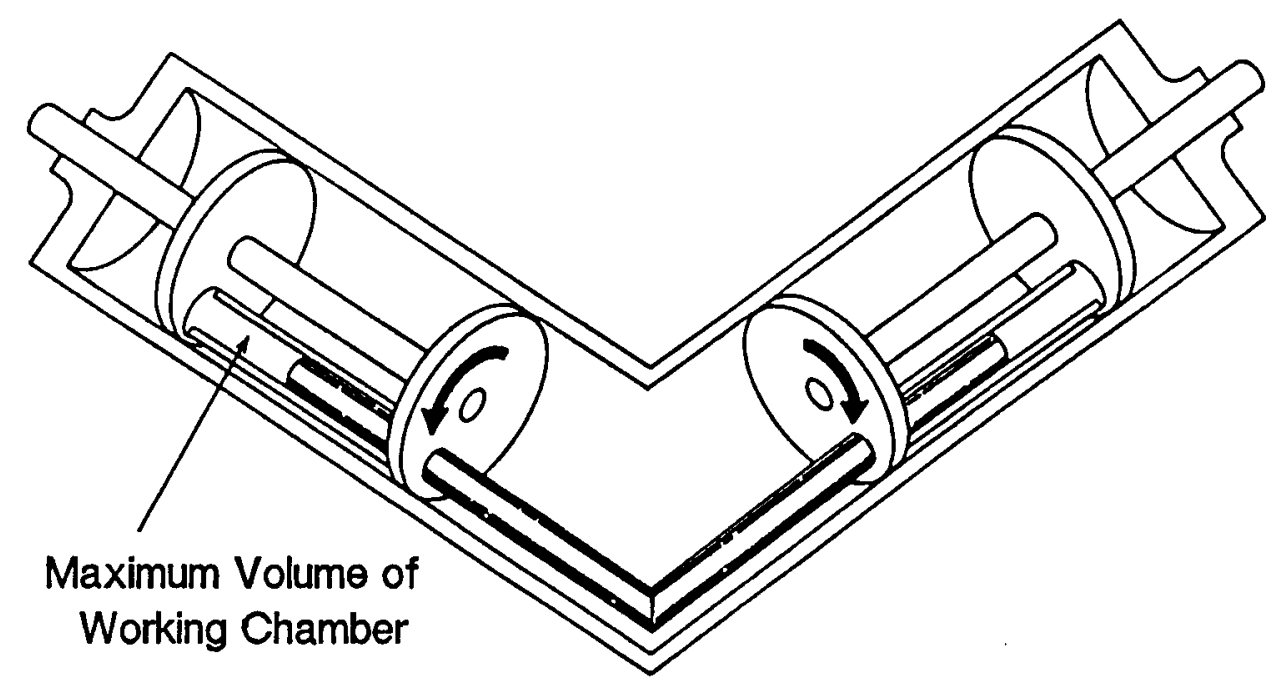

Figure 4 -- The Rotary-Vee Engine With the Piston at BDC 


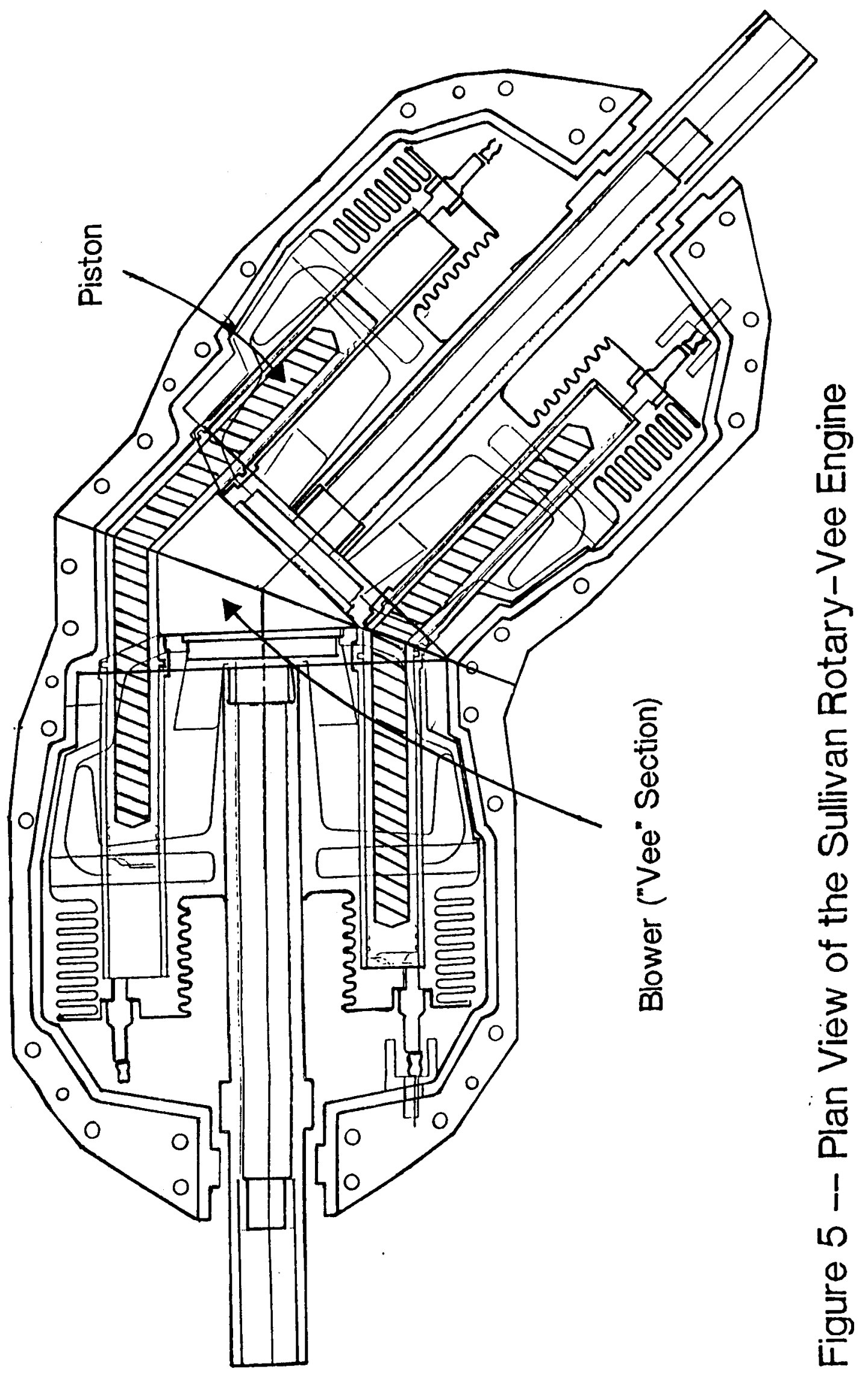



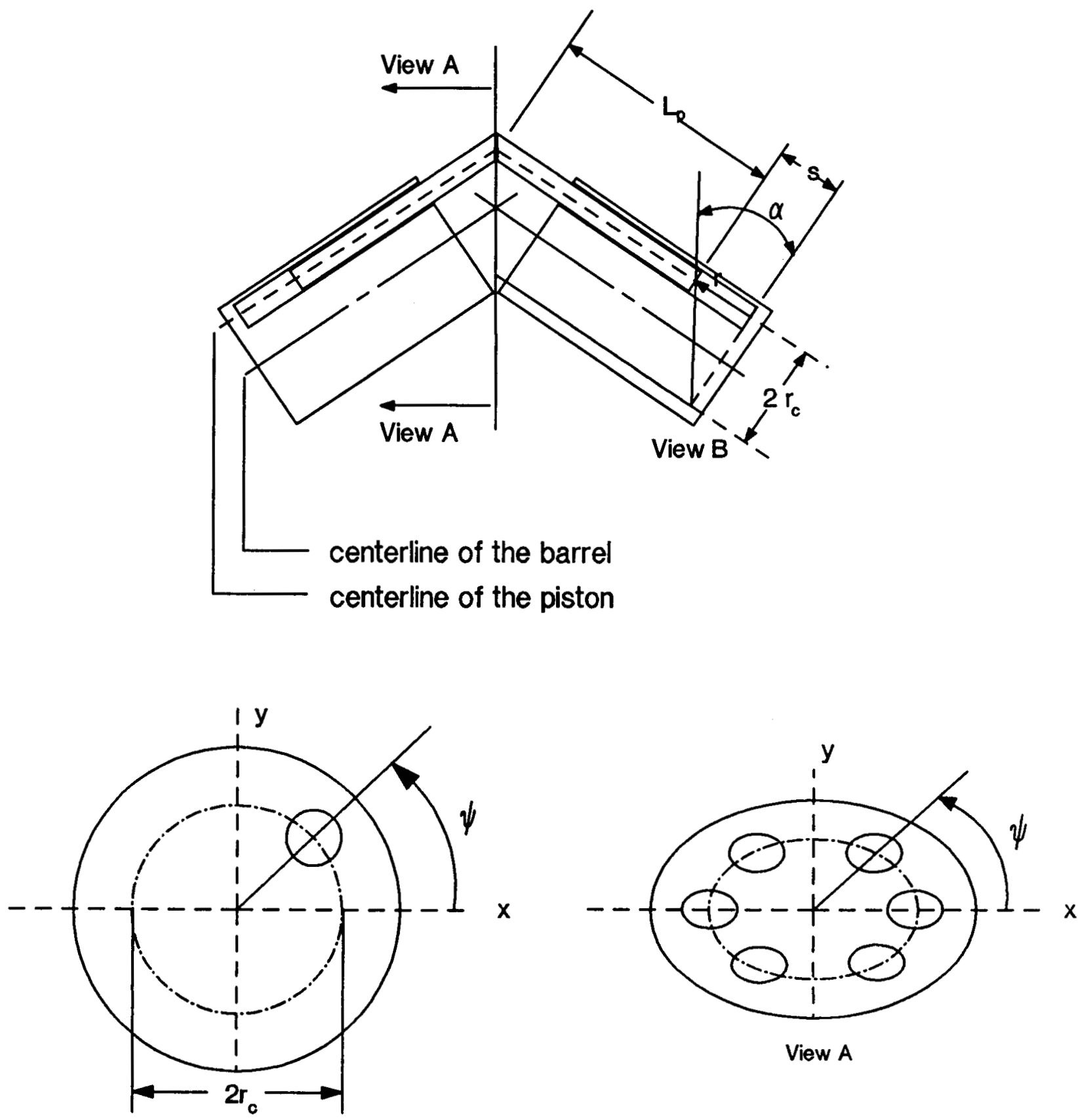

View B

Figure 6 -- Rotary-Vee Geometry 

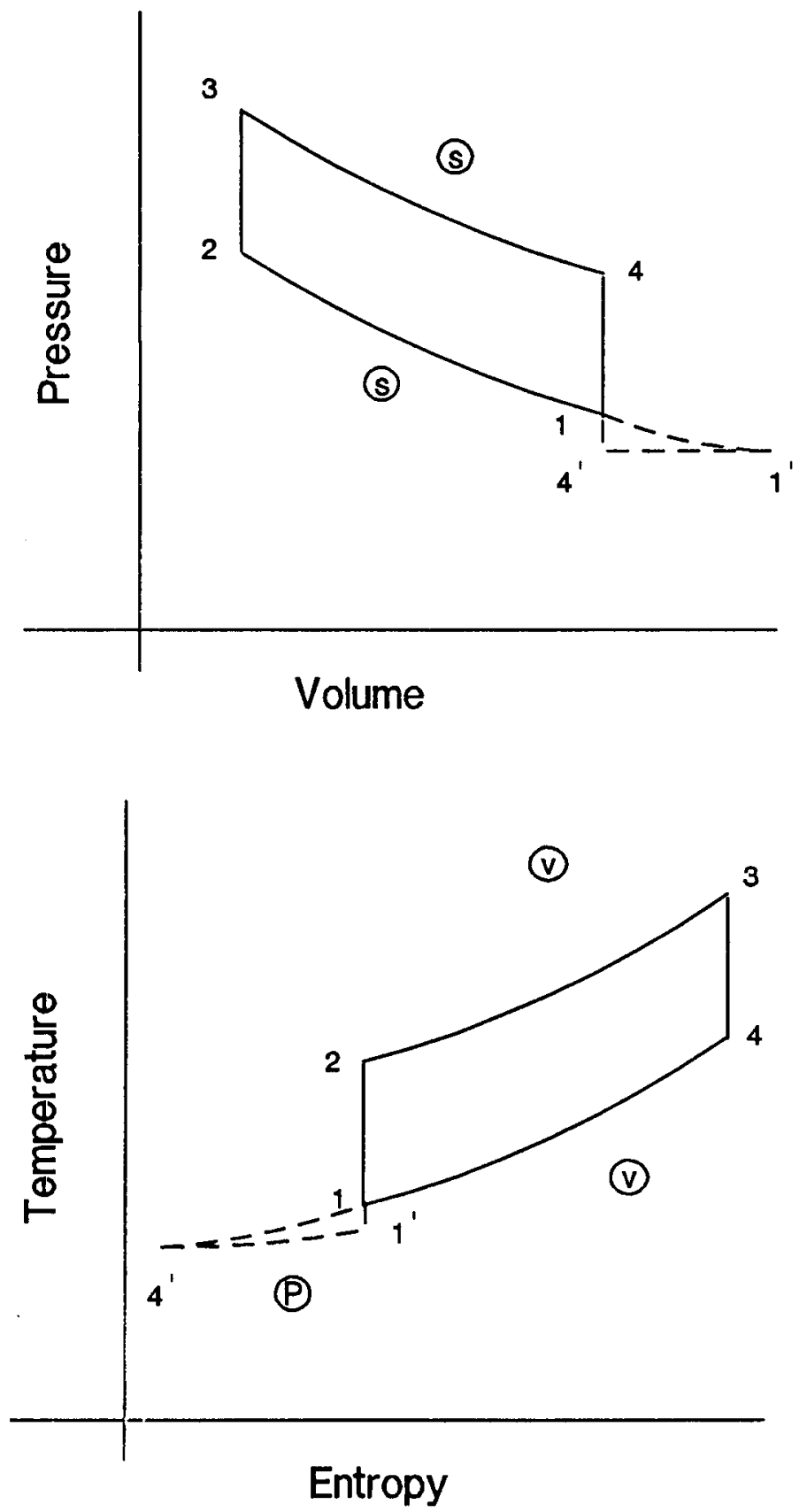

Figure 7 -- Pressure-Volume and Temperature-Entropy Diagrams for Rotary-Vee Engine 

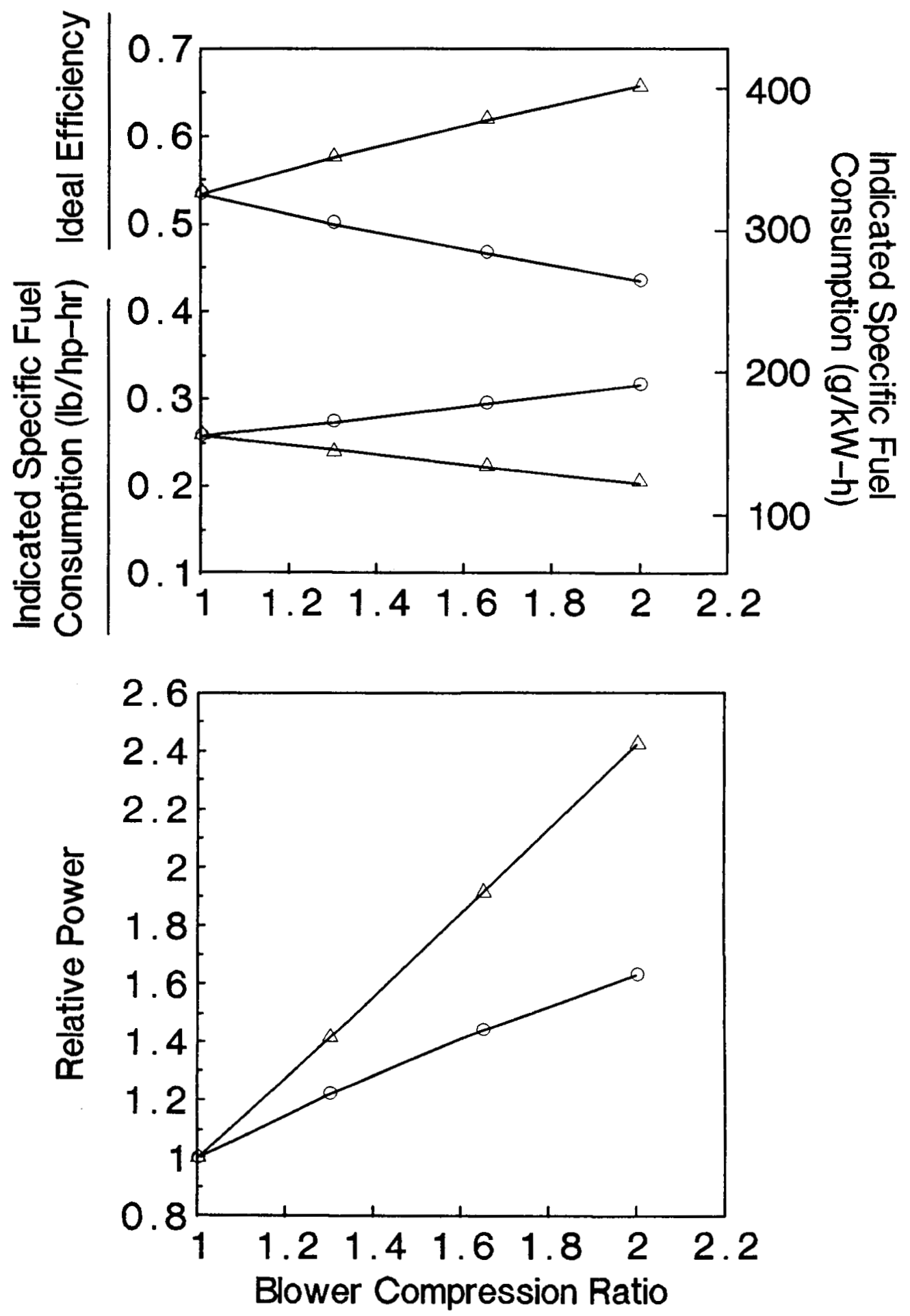

$\circ$ Rotary-Vee $\triangle$ Full Expansion

Figure 8 -- Performance Comparison of Rotary-Vee and Full Expansion Cycles 


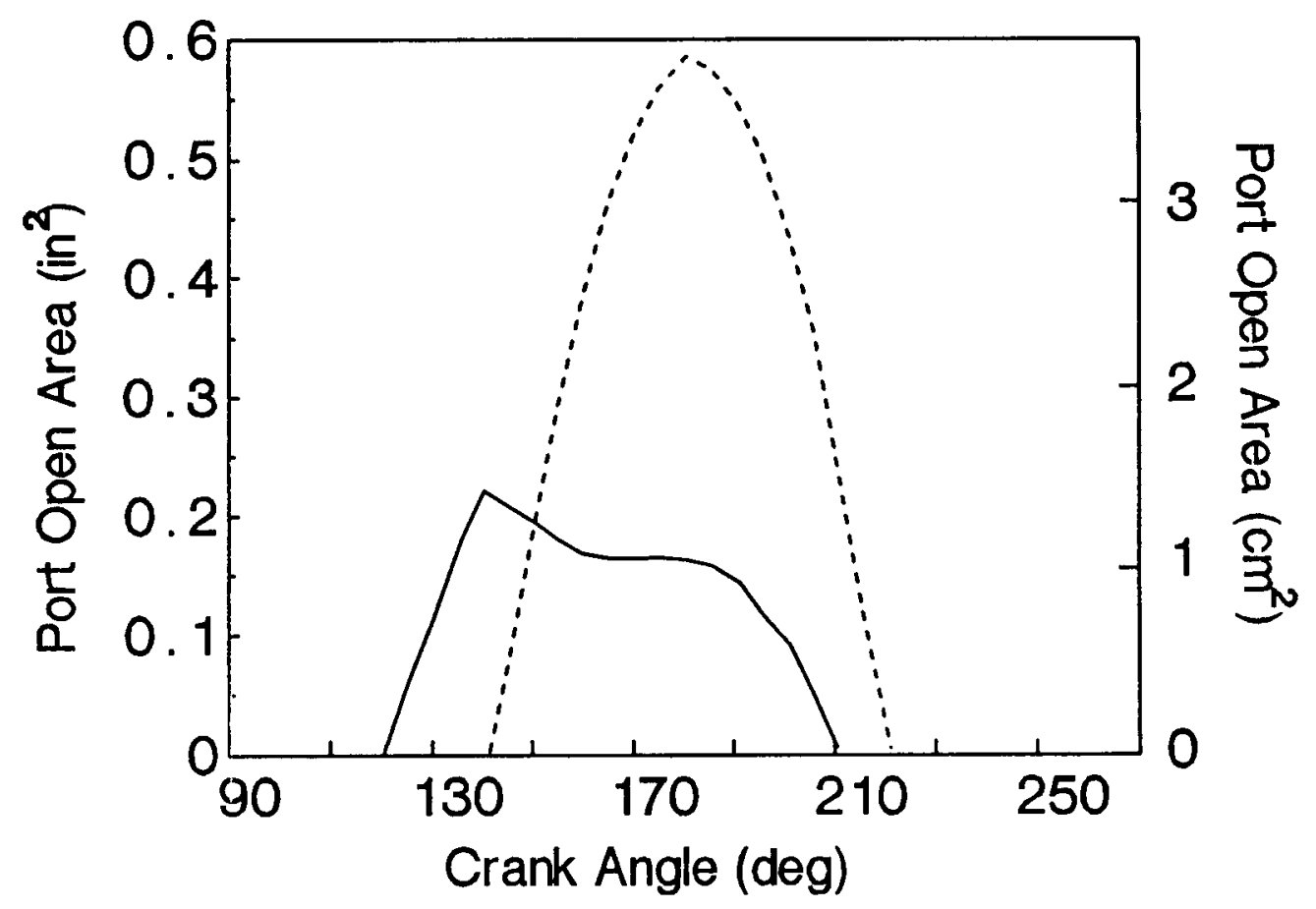

...- Intake Port - Exhaust Port

Figure 9 -- Instantaneous Port Open Areas

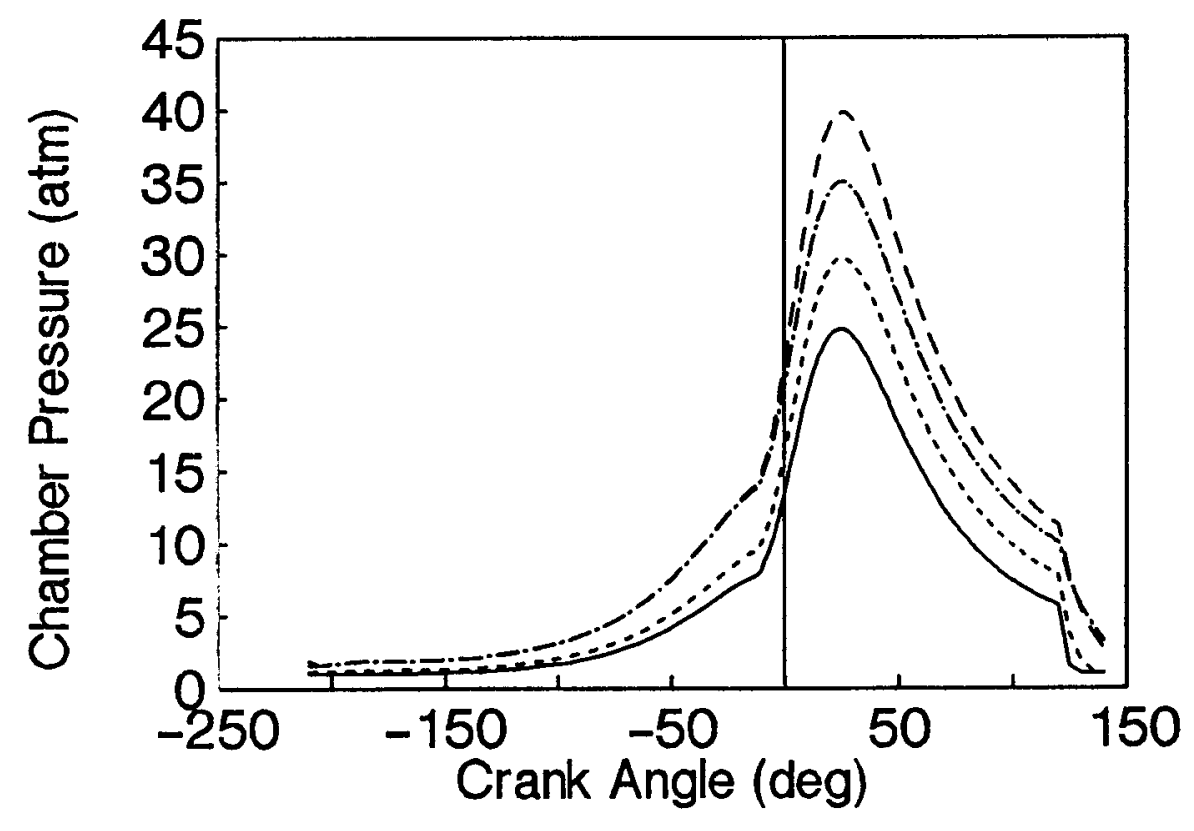

\section{- $1250 \mathrm{rpm} \quad \cdots 2500 \mathrm{rpm} \quad--5200 \mathrm{rpm}$}

$-.-7000 \mathrm{rpm}$

Figure 10 -- Instantaneous Chamber Pressure for the Rotary-Vee Engine at Various Speeds (Spark Advance, $\theta_{0}=15^{\circ} \mathrm{BTDC}$ ) 


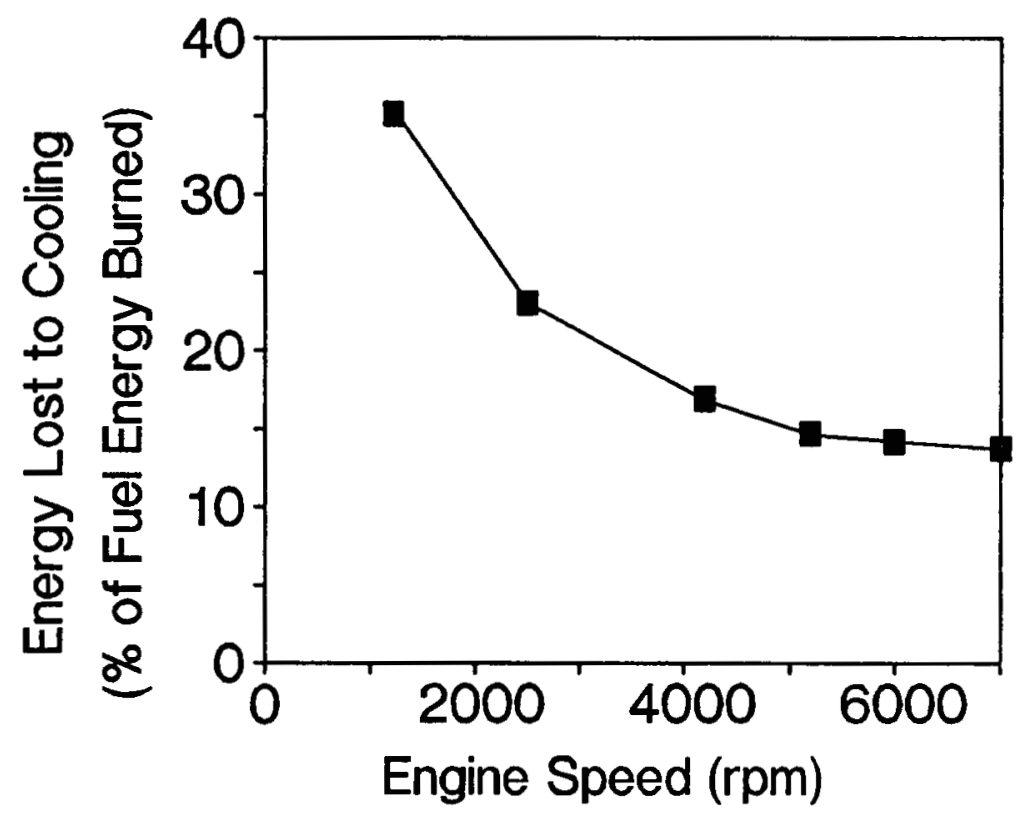

Figure 11 -- Fuel Energy Lost to Coolant for Various Engine Speeds

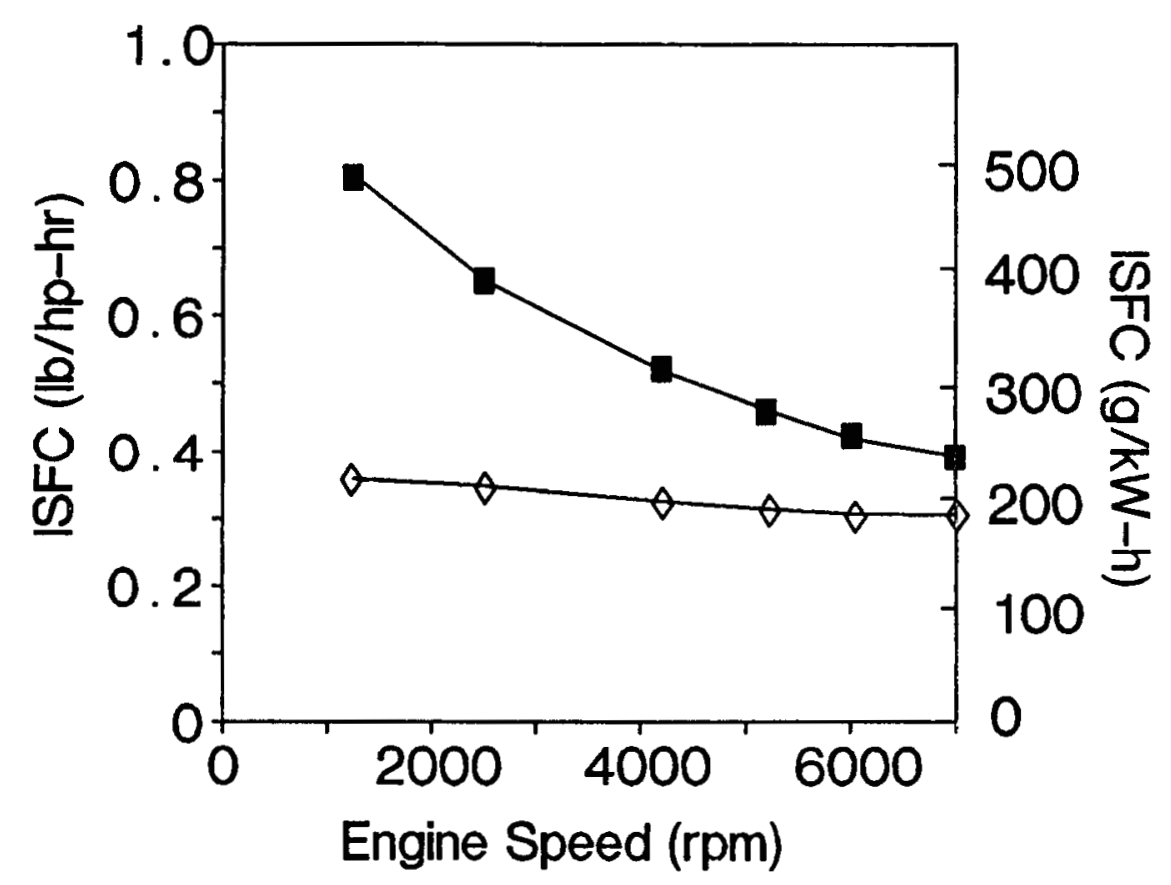

- Carbureted $\diamond$ Direct Cylinder

Fuel Injection

Figure 12 -- Indicated Specific Fuel Consumption v. Engine Speed 


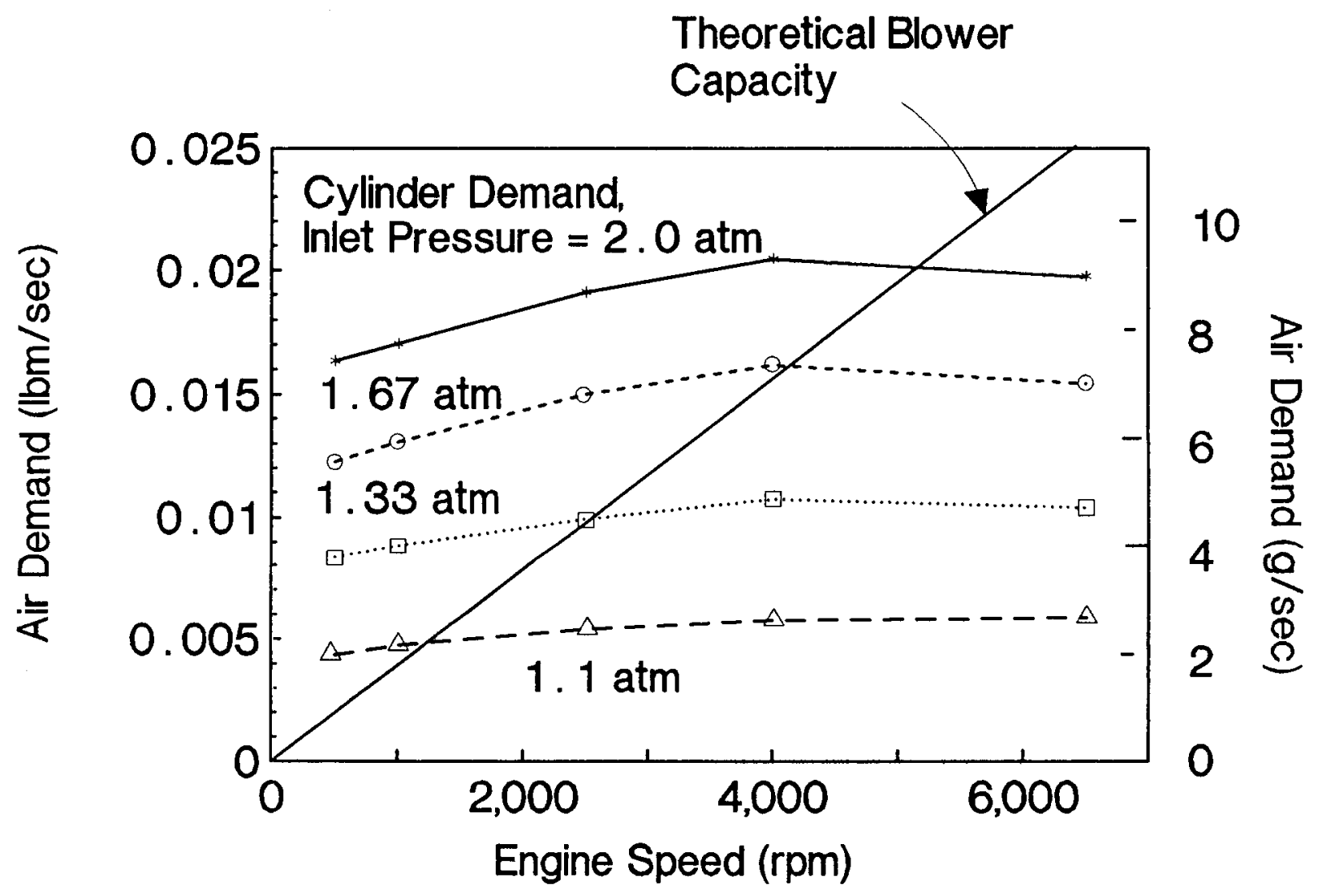

Figure 13 -- Air Mass Flow Rate vs. Engine Speed for Various Blower Outlet Pressures 


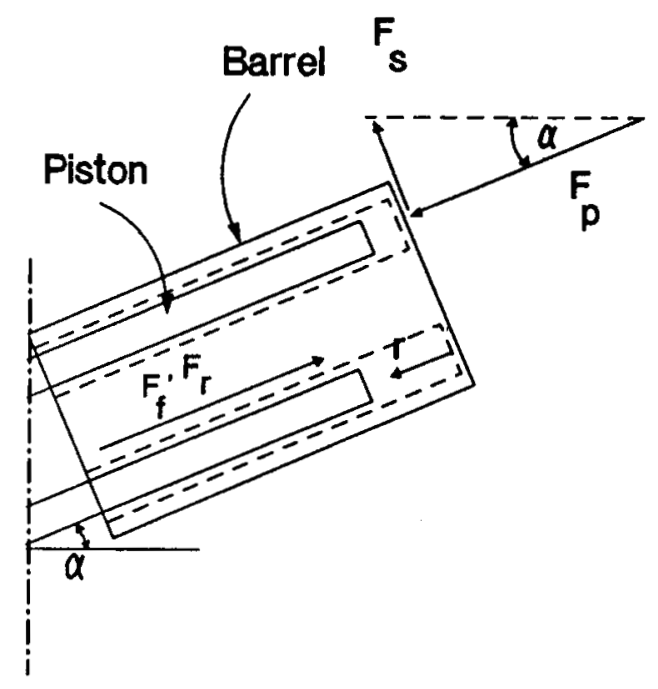

(a) Half Rotary-Vee, in the "V-plane"

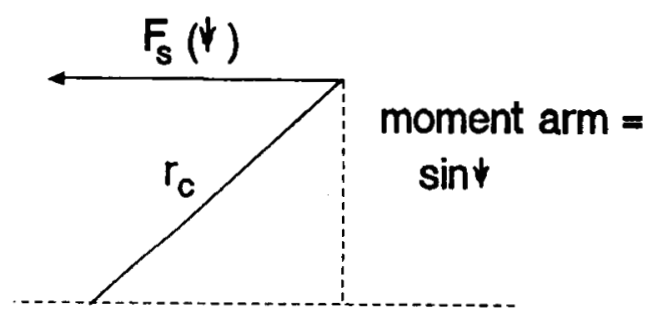

(c) Indicated Torque

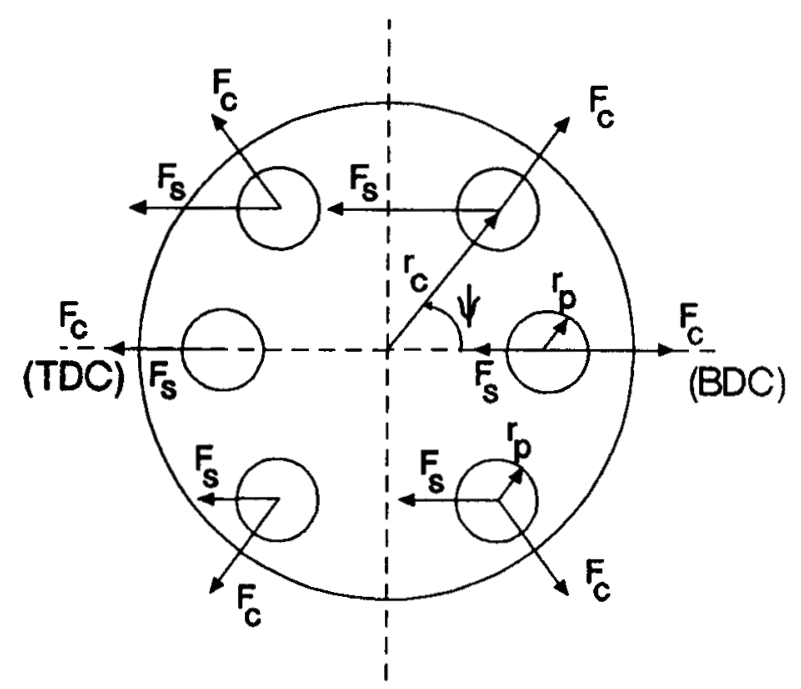

(b) Barrel Cross Section

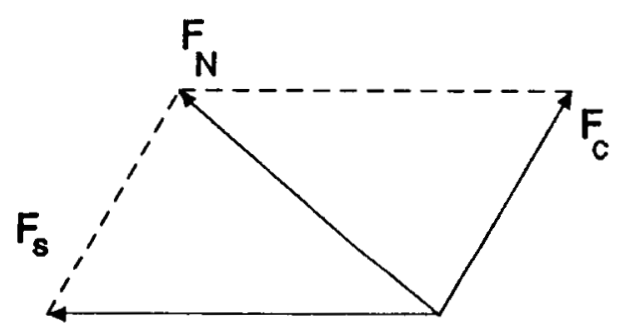

(d) Normal Force on the Sleeve

Figure 14 -- Elements of Kinematic Analysis for the Rotary-Vee 


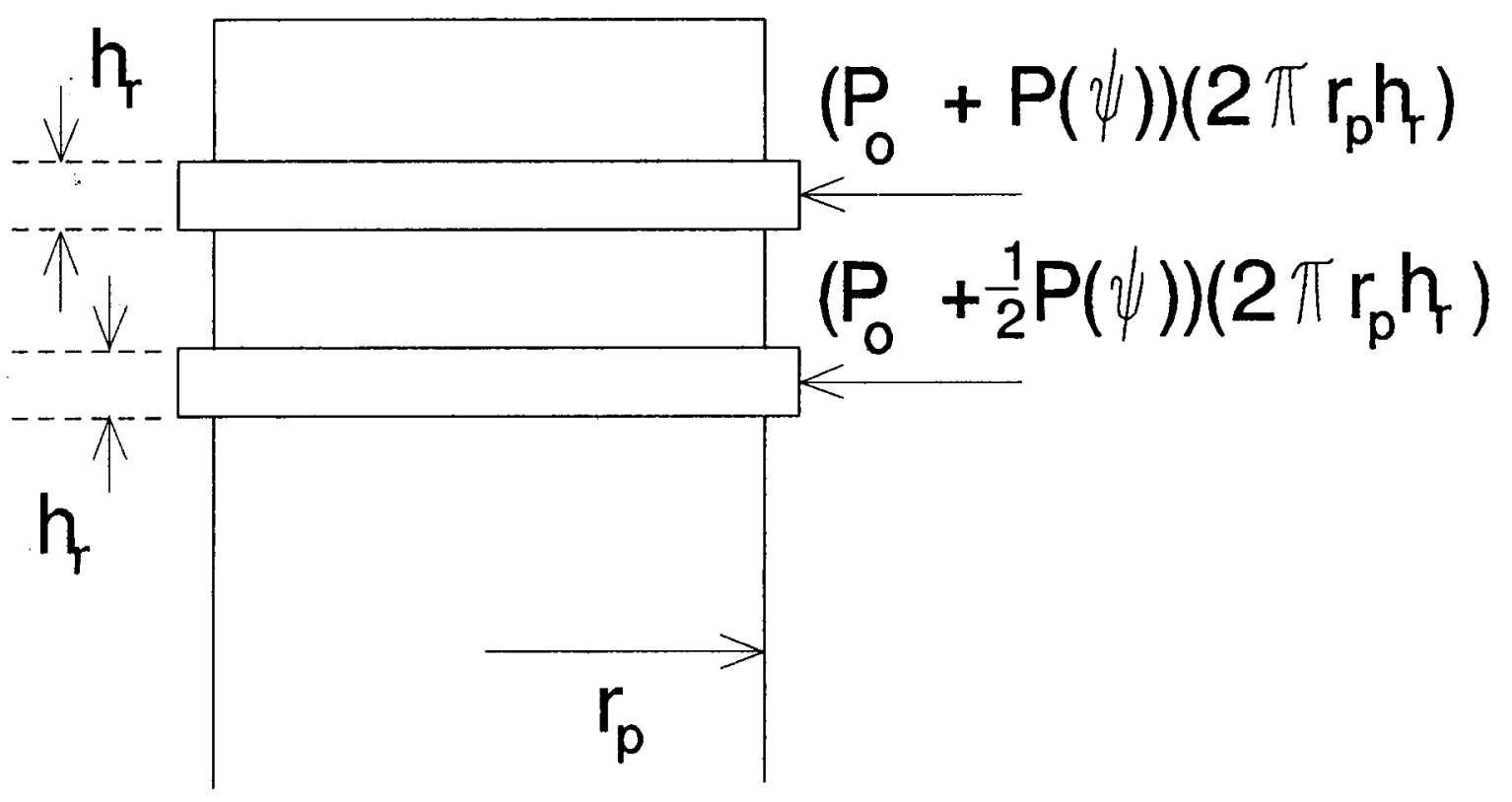

Figure 15 -- Ring Pack Geometry and Forces 

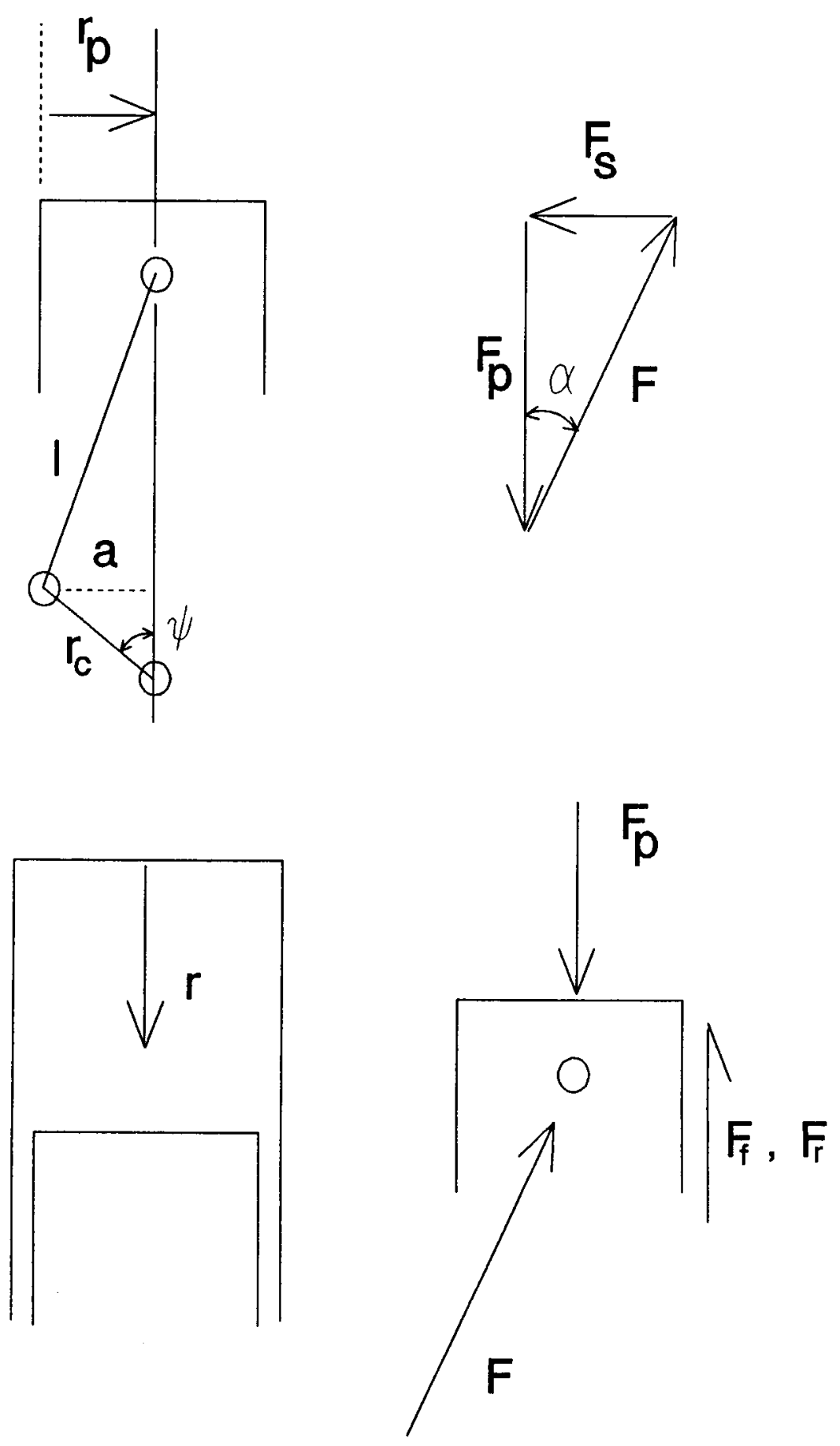

Figure 16 -- Geometry of Crank Piston Engine 


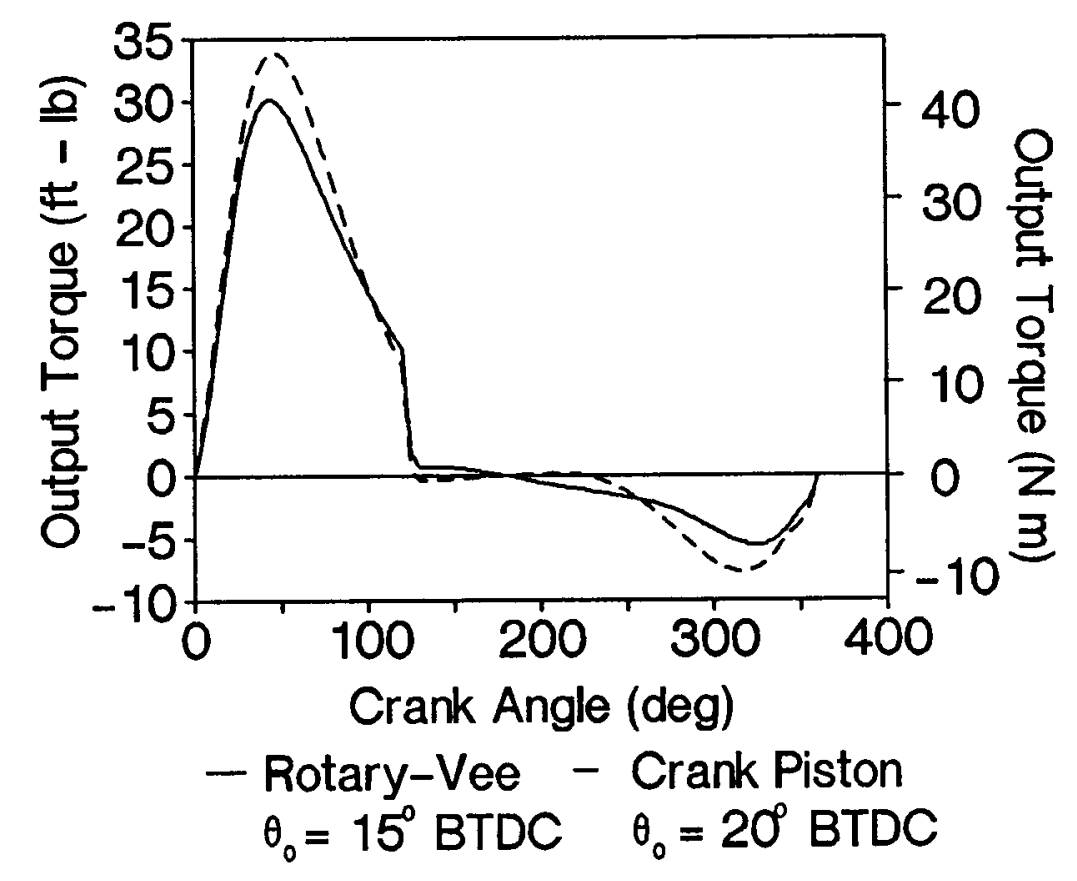

(a) $1250 \mathrm{rpm}$

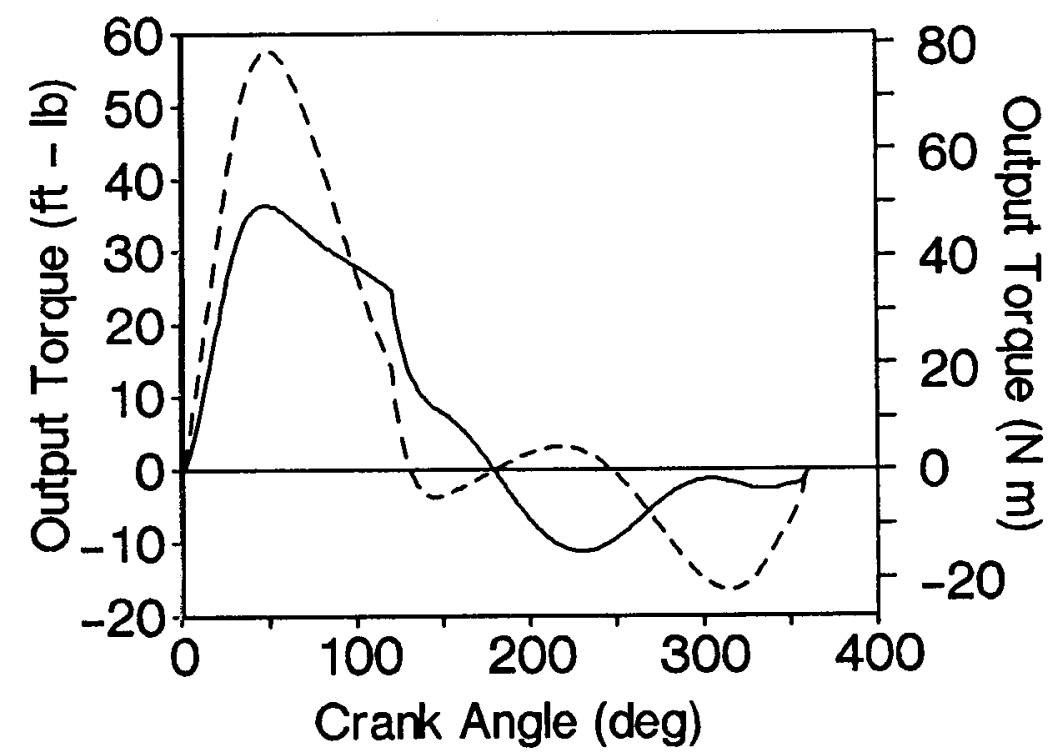

- Rotary-Vee - Crank Piston $\theta_{0}=15^{\circ}$ BTDC $\theta_{0}=20^{\circ}$ BTDC

(b) $4200 \mathrm{rpm}$

Figure 17 -- Instantaneous Output Torque (Neglecting Blower and Cooling Losses) for the Rotary-Vee and Crank Piston Engines 


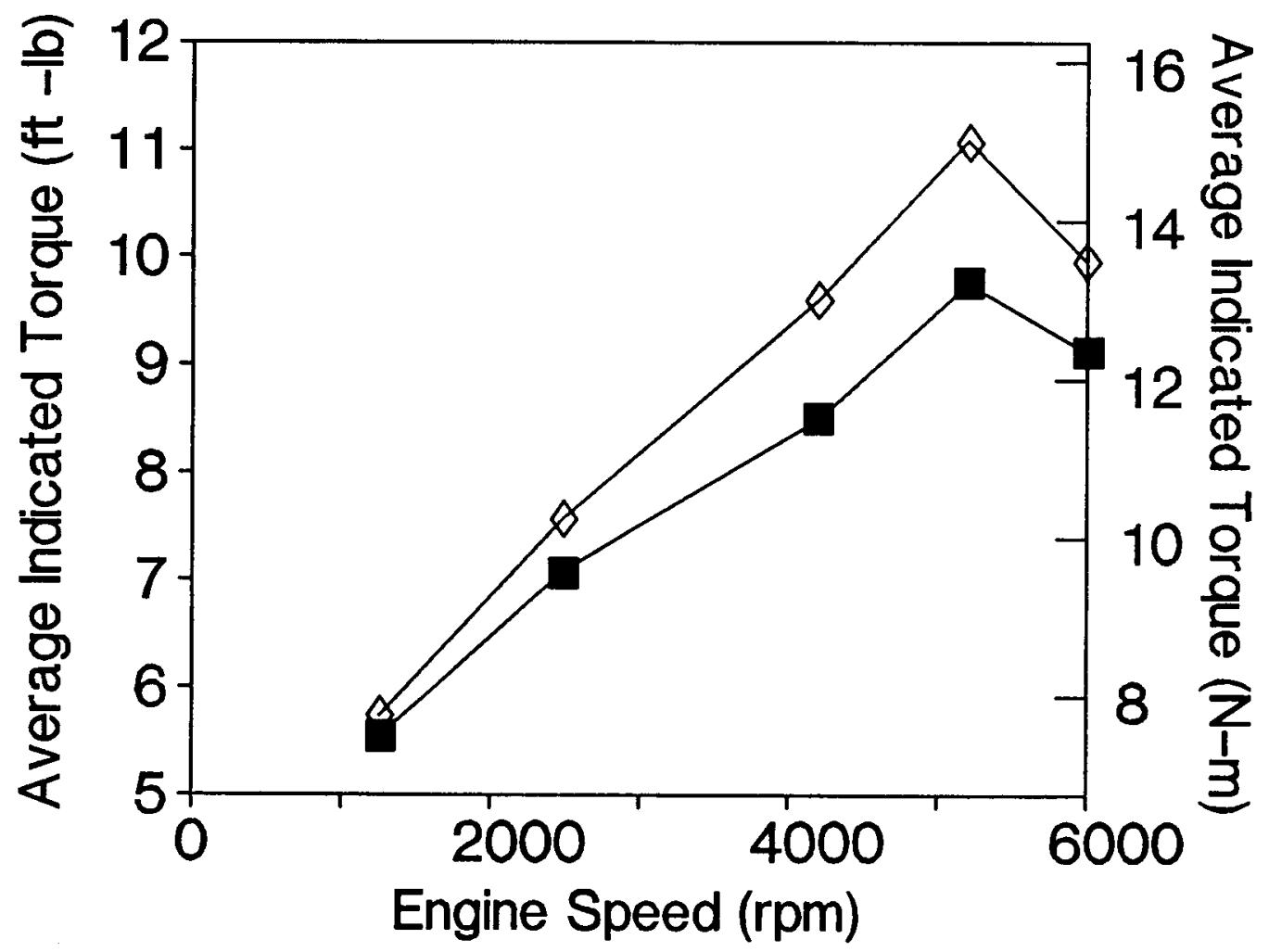

- Rotary-Vee (Spark Fires at 15 deg BTDC)

$\diamond$ Crank Piston (Spark Fires at 20 deg BTDC)

Figure 18 -- Average indicated Torque per Cylinder v. Engine Speed (Blower and Cooling Losses not Included) 


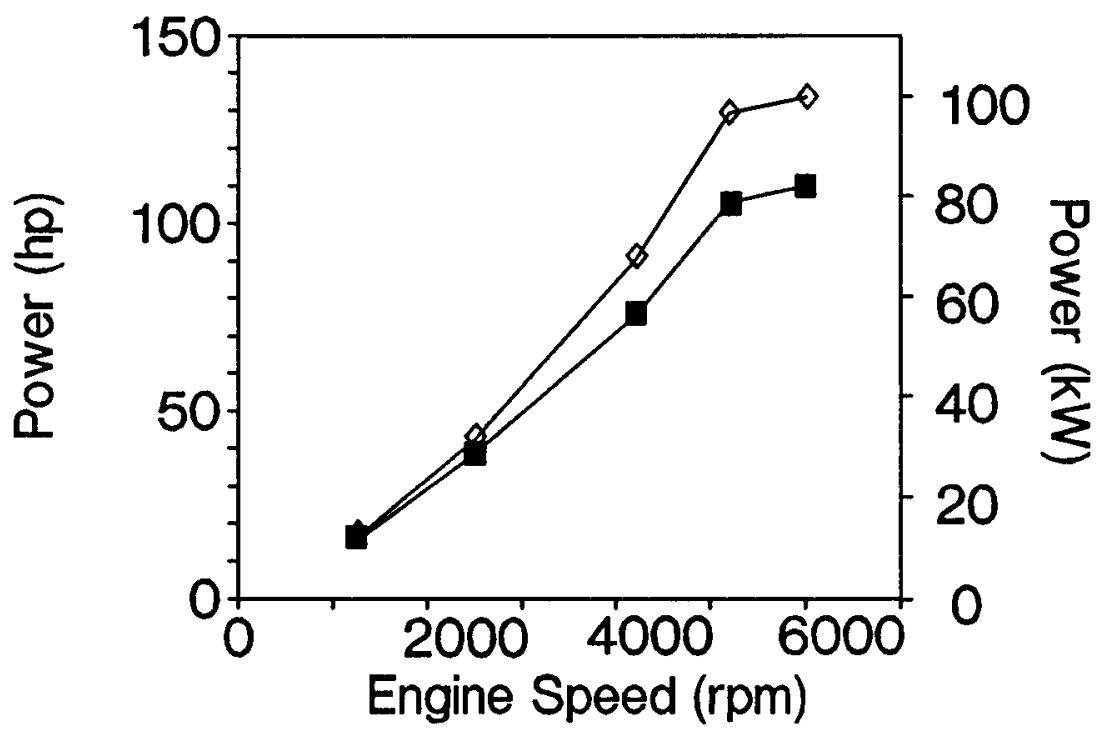

- Rotary-Vee

(Spark at 15 deg BDTC)

$\diamond$ Crank Piston

(Spark at 20 deg BTDC)

(a) Output Power

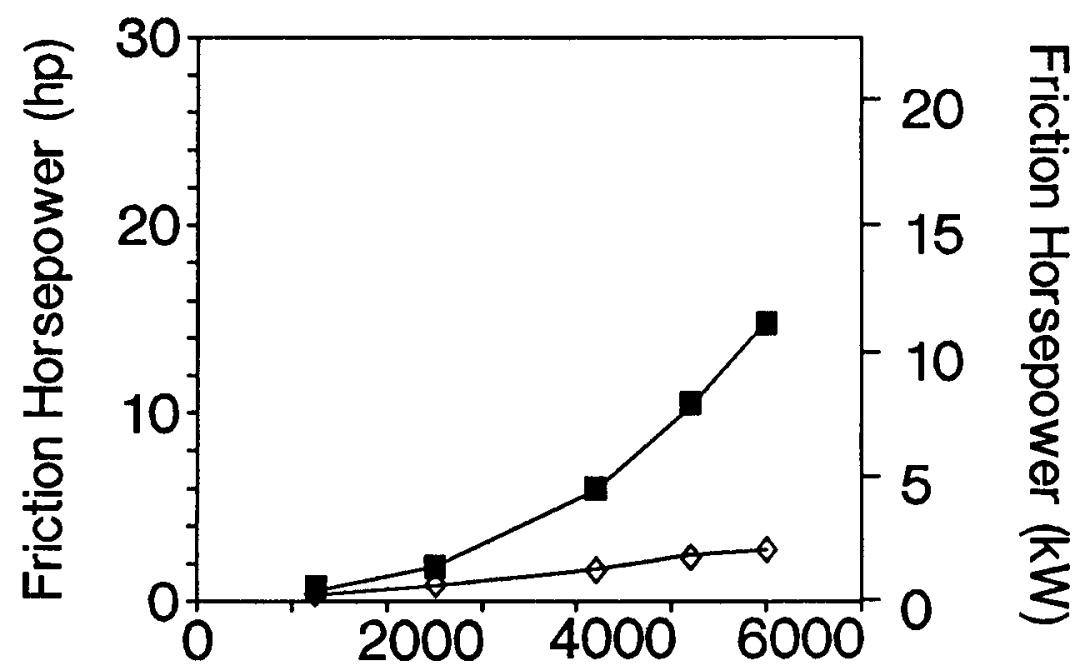

Engine Speed (rpm)

- Rotary-Vee

(Spark at 15 deg BDTC) $\diamond$ Crank Piston

(Spark at 20 deg BTDC)

(b) Friction Power

Figure 19 -- Output Horsepower (Blower and Cooling Losses Not Included) and Friction Hordepower for the Rotary-Vee and Crank Piston Engines 


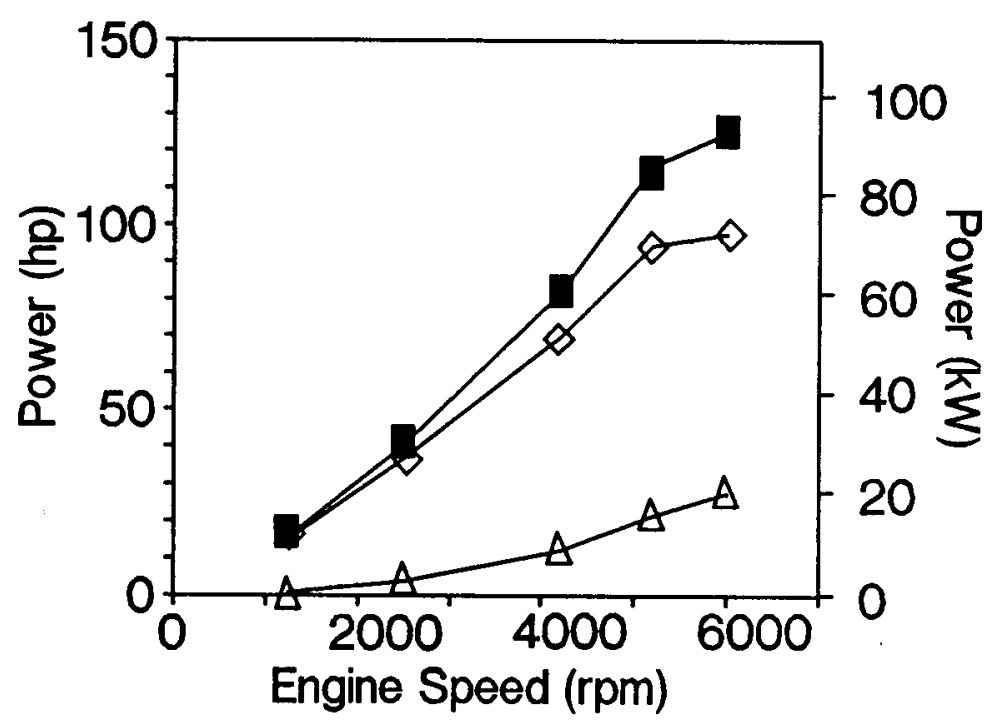

- inp $\diamond$ bhp $\triangle$ Sum of the Parasitic Losses

(a) Rotary-Vee Output

(Spark Advance, 15 deg BTDC)

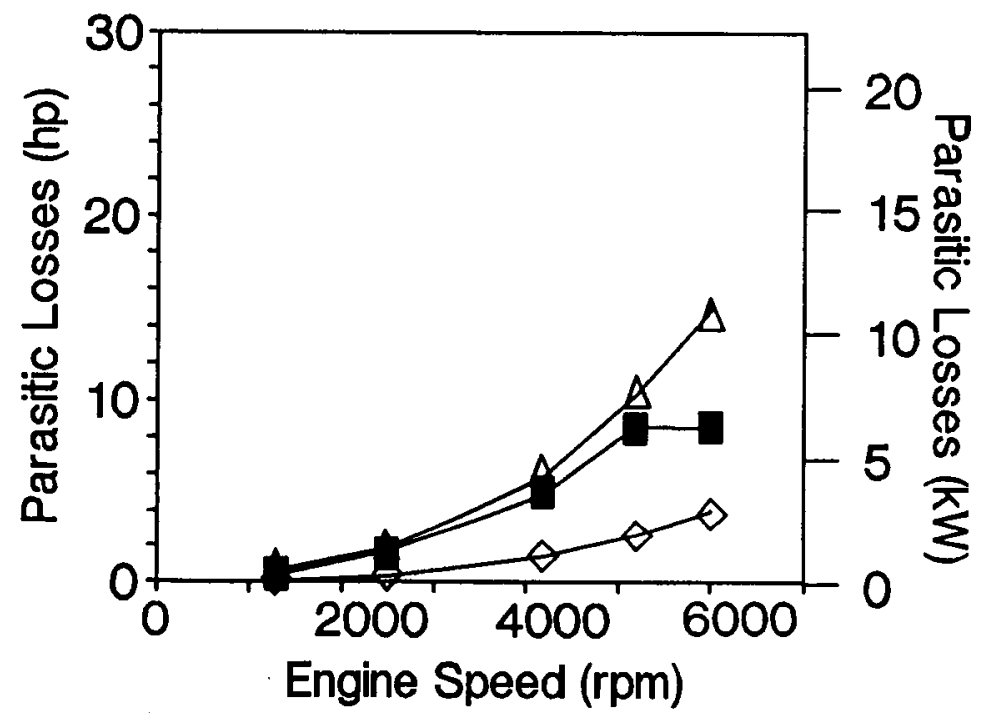

- Blower Losses $\diamond$ Fan Losses

$\triangle$ Friction Losses

(b) Parasitic Losses

(Spark Advance, 15 deg BTDC)

Figure 20 -- Rotary-Vee Performance and Fuel Consumption 


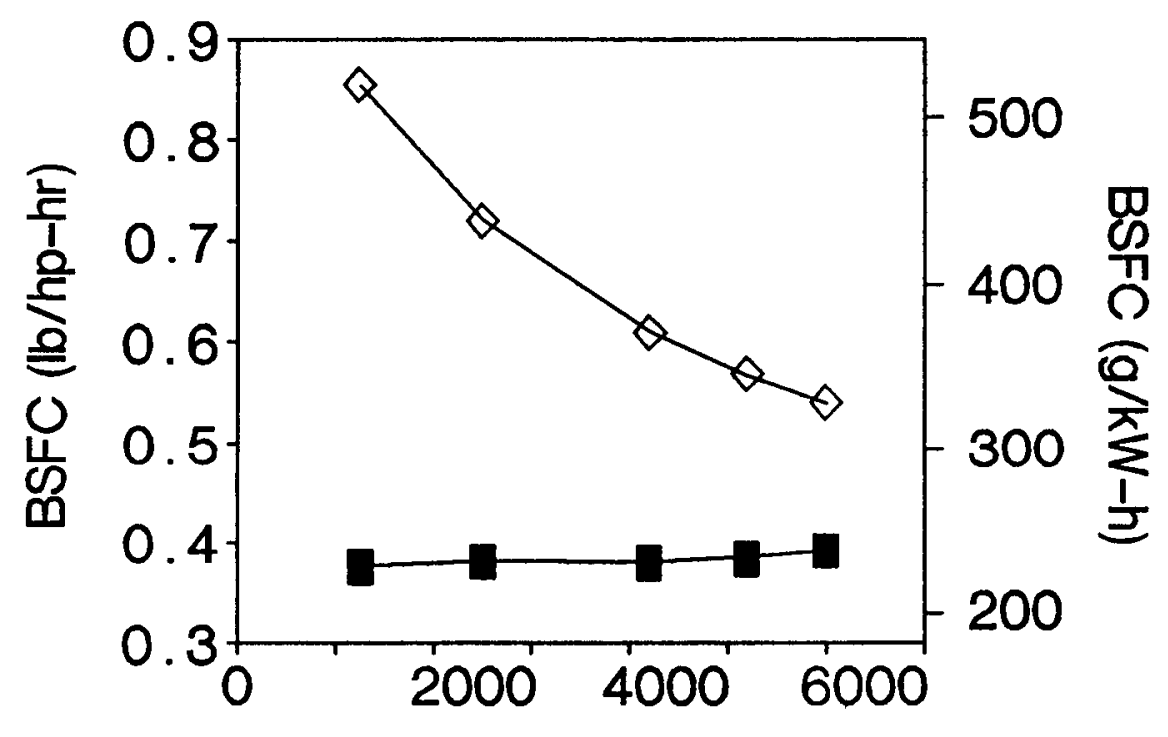

Engine Speed (rpm)

- Direct Cylinder $\diamond$ Carbureted Fuel Injection

(c) BSFC

(Spark Advance, 15 deg BTDC)

Figure 20 -- (Continued) 


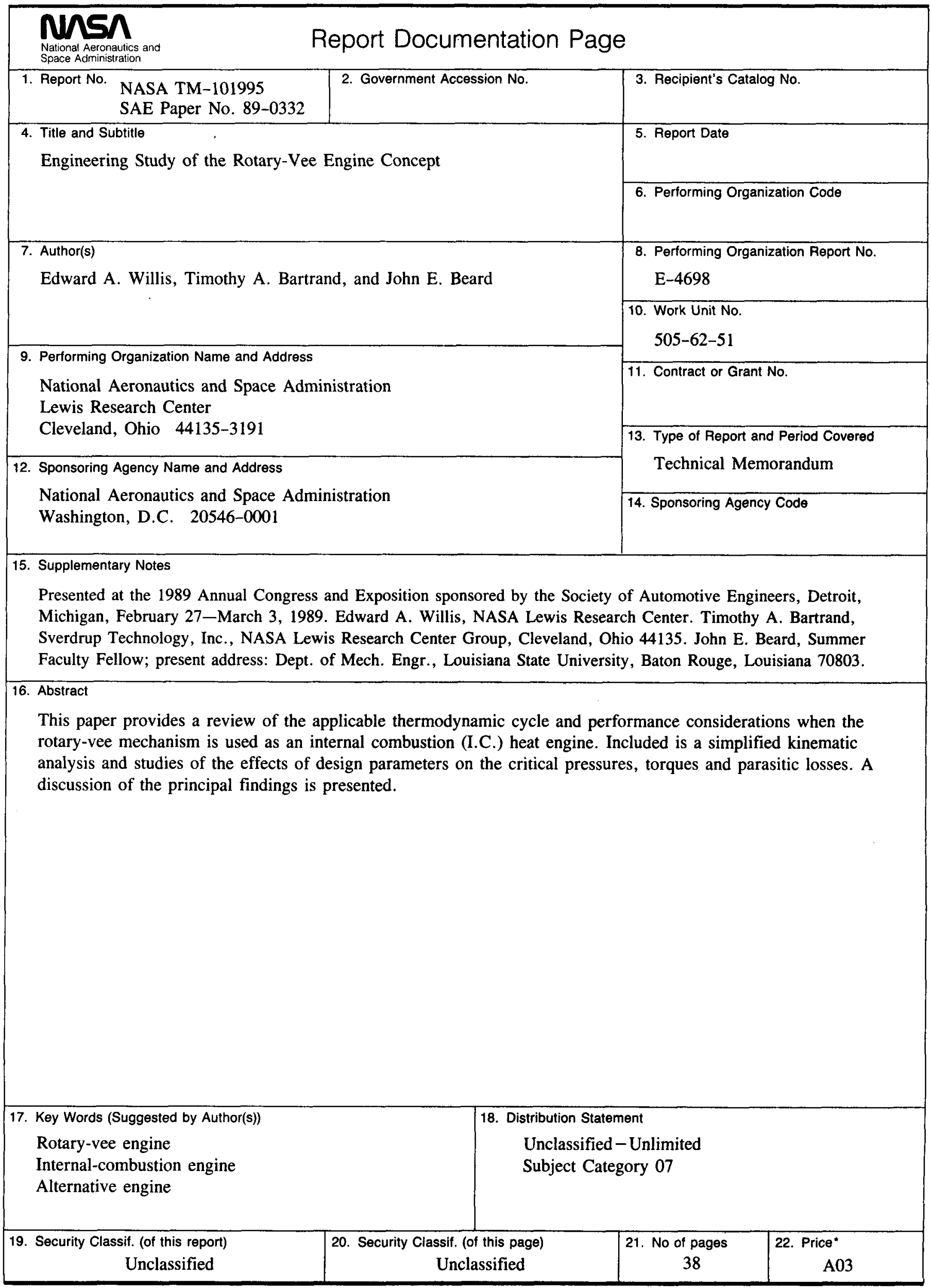

